\title{
EFFECTS OF VISCOUS DISSIPATION ON MISCIBLE THERMO-VISCOUS FINGERING INSTABILITY IN POROUS MEDIA
}

\author{
M. Norouzi ${ }^{1}$, S. Dorrani ${ }^{1}$, H. Shokri ${ }^{1}$ and O. Anwar Bég ${ }^{2}$ \\ ${ }^{l}$ Faculty of Mechanical Engineering, Shahrood University of Technology, Shahrood, Iran. \\ Fluid Mechanics, Department of Mechanical/Aeronautical Engineering, Salford University, Manchester, M54WT, UK.
}

\begin{abstract}
The thermo-viscous fingering instability associated with miscible displacement through a porous medium is studied numerically, motivated by applications in upstream oil industries especially enhanced oil recovery (EOR) via wells using hot water flooding and steam flooding. The main innovative aspect of this study is the inclusion of the effects of viscous dissipation on thermal viscous fingering instability. An Arrhenius equation of state is employed for describing the dependency of viscosity on temperature. The normalized conservation equations are solved with the finite element computational fluid dynamics code, COMSOL (Version 5) in which glycerol is considered as the solute and water as the solvent and the two-phase Darcy model employed (which couples the study Darcy flow equation with the time-dependent convection-diffusion equation for the concentration). The progress of finger patterns is studied using concentration and temperature contours, transversely averaged profiles, mixing length and sweep efficiency. The sweep efficiency is a property widely used in industry to characterize how effective is displacement and it can be defined as the ratio of the volume of displaced fluid to the total volume of available fluid in a porous medium in the displacement process. The effects of Lewis number, Brinkman number and thermal lag coefficient on this instability are examined in detail. The results indicate that increasing viscous dissipation generates significant enhancement in the temperature and a marked reduction in viscosity especially in the displaced fluid (high viscous phase). Therefore, the mobility ratio is reduced, and the flow becomes more stable in the presence of viscous dissipation.
\end{abstract}

Keywords: Thermal viscous fingering instability; Viscous dissipation; Porous media; COMSOL; Computational fluid dynamics; Time-dependent convection-diffusion equation; Mixing length.

*Corresponding Author, Associate Professor in Mechanical Engineering, Phone: (+98)

9123726933, Fax: (+98) 2733300258, email: manorouzi@shahroodut.ac.ir

\section{INTRODUCTION}

Saffman-Taylor instability is one of the well-known hydrodynamic instabilities which arises in the displacement of fluids through porous media. This instability is observed when a high 
viscosity fluid is displaced by a low viscosity one. This instability actually should be referred to as the "Hill instability" since the chemical engineer Hill [1] was historically the first to identify and analyze this phenomenon. Indeed, the viscous mismatch at the interfaces between fluids involved in displacement is the main factor behind this instability. On other hand, this instability manifests itself in the form of finger-shaped penetrations of the displacing fluid into the displaced one. Consequently, it is also referred to as viscous fingering instability and is observed in a wide variety of industrial processes including enhanced oil recovery (EOR), filtration, packed bed regeneration, geothermal reservoir recharge and electrochemical deposition. In most applications, this instability is an undesirable phenomenon since it reduces the sweep efficiency which in turn causes a significant reduction in, for example, enhanced oil recovery processes $[1,2]$. Therefore, any methodology which can lead to the elimination or mitigation of this instability or control of its growth rate is technically important. This goal has led to a large amount of studies that focus on both theoretical and computational analysis of instability.

The seminal work concerning Saffman-Taylor instability has been presented by Hill [3]. An excellent review of viscous fingering in permeable media (of relevance to petroleum engineering) has also been produced by Homsy [3]. Since then, numerous other investigations have been presented in the scientific literature. These studies can be classified into two categories: isothermal or non-isothermal flows. Indeed, a change in the flow temperature leads to variation in viscosity which is a main characteristic of fingering instability. However, the vast majority of studies have been restricted to isothermal fingering instability.

Tan and Homsy $[4,5]$ studied the stability of isothermal miscible rectilinear and radial displacement processes in porous media. They used the quasi-steady-state approximation to predict accurately the growth rate of disturbances. Singh and Azaiez $[6,7]$ investigated the viscous fingering in miscible displacement, considering shear-thinning fluids using both a 
pseudo-spectral numerical technique based on the Hartley transform and a linear stability analysis. In addition to the patterns already known for purely Newtonian flow displacements, several new mechanisms were observed in their nonlinear simulations. Their results of linear stability analysis also identified that a flow where the displacing fluid is shear-thinning is always more unstable than its Newtonian counterpart. More recently, Norouzi and Shoghi [8] examined the effect of anisotropic porous media on viscous fingering in miscible displacements. Their results demonstrated that the flow is more stable for larger values of the anisotropic permeability ratio and also for smaller values of anisotropic dispersion ratio. Shoghi and Norouzi [9] also simulated miscible displacements in the presence of permeability heterogeneity for a displacing shear-thinning fluid, noting that the differences between Newtonian and non-Newtonian flows are amplified for higher degrees of heterogeneous porous media. The viscous fingering instability of miscible displacement involving a viscoelastic fluid was also investigated by Shokri et al. $[10,11]$. They found that the elasticity of viscoelastic fluid has a stabilizing effect on the flow field. Their results also showed that the flow becomes more stable by increasing the permeability of porous media in the longitudinal direction relative to the transverse direction.

In addition to the vast amount of studies which have considered the isothermal fingering instability, a few studies addressing non-isothermal fingering instability have also been communicated in the literature. The viscosity of fluids, the main factor behind the fingering instability, is a function of temperature. Consequently, the difference in temperature of two fluids involved in displacement flows can have a significant influence on fingering instability. Under such conditions, the instability is called thermo-viscous fingering. In miscible displacement, the concentration and thermal diffusion rates may also differ from one another. Therefore, there are two fronts in the flow: the thermal front and the concentration front. The thermal front is usually slower than concentration front due to heat transfer between fluids and 
porous media. The thermal front therefore invariably lags behind the concentration front. The thermo-viscous fingering instability can be seen in many petro-chemical engineering processes such as hot water flooding, steam flooding and Steam Assisted Gravity Drainage (SAGD).

The pioneering study in thermo-viscous fingering instability was reported by Kong et al. [12]. They experimentally investigated the displacement of both synthetic (Dutrex 739) and natural heavy oils by steam under various conditions in vertical and horizontal rectilinear HeleShaw cells. Saghir et al. [13] studied two-dimensional nonlinear double diffusive convection in a multi-porous cavity, both numerically and experimentally. They deployed glycerin and water in their experiments and observed that the difference between the tip and the base of the displacement front decreases when hot water is injected into the system. Their results further showed that the buoyancy force opposed the growth of fingers. Sheory et al. [14] presented a numerical reservoir simulation model for the study of enhanced oil recovery. Their results show that oil recovery can be improved when the formation temperature is higher, or both the injection temperature and pressure are raised. Holloway and Bruyn [15] studied the fingering instability for the scenario in which hot glycerin displaces cold more viscous glycerin in a radial Hele-Shaw cell and concluded that the wavelength of the fingering instability is proportional to the cell width for thin cells. An experimental study of the displacement of a high-viscosity fluid at low temperature by the same fluid at high temperature and with low viscosity in cylindrical capillary tubes has been presented by Kuang and Maxworthy [16]. They studied three different regimes in their work: the diffusion dominated regime, viscously dominated regime and transition regime. A linear stability analysis for viscous fingering instability of the double-front system was presented by Pritchard [17] in which it was found that the properties of each front (thermal or mass front) contribute to the tendency of the system to become unstable. Islam and Azaiez [18, 19] studied the thermo-viscous fingering instability in miscible displacement in a Hele-Shaw cell using both linear stability analysis and numerical simulation. 
Their results show that an increase in thermal mobility ratio leads to more unstable flow. Additionally, a decrease in thermal-lag coefficient decreases the instability. Azaiez and Sajjadi [20] presented a paradigm on the stability of two-component miscible displacement in a homogeneous porous medium. In their study, the components had different mobility ratios, were able to diffuse at different rates and convected at different speeds. Sajjadi and Azaiez [21] also investigated the non-isothermal miscible viscous flow via nonlinear simulation. They focused on the effect of heat transfer between the displacing fluid and the solid matrix on the thermo-viscous instability. Their results indicated that the rate of heat transfer with the medium is a significant factor contributing to this instability. More recently, Jackson et al. [22] investigated the immiscible radial displacement in a Hele-Shaw cell with a temperaturedependent viscosity using two coupled high resolution numerical methods (i.e. an auxiliary radial basis function-finite collocation (RBF-FC) method and a boundary element - RBF-FC method) .

In the present paper, the miscible thermo-viscous fingering instability in porous media is studied numerically with COMSOL multi-physics computational fluid dynamic (CFD) commercial software. Viscous dissipation effects are included since this important phenomenon characterizing real flows has been omitted in previous works. The viscosity of most liquids is severely decreased by increasing the temperature and furthermore viscous dissipation is also directly dependent on the viscosity. Therefore, it is anticipated that the viscous dissipation could markedly influence the thermo-viscous fingering instability. In the simulations, it is assumed that the two fluids are miscible and the porous media is homogenous. An Arrhenius equation of state for describing the dependency of viscosity on temperature is implemented. The effects of Brinkman number, Lewis number and thermal lag coefficient on this instability are studied in detail. The concentration and temperature contours, transversely averaged profiles, mixing length and sweep efficiency are computed for different scenarios 
with viscous dissipation. The mechanisms of growth of the fingers under different flow conditions are also discussed.

\section{GOVERNING EQUATIONS}

In this study, miscible displacement in a homogenous porous medium is studied. A hot lowviscosity fluid $\left(\mu_{1}, T_{1}\right)$ displaces a cold high viscosity liquid $\left(\mu_{2}, T_{2}\right)$. The governing equations comprise the conservation of mass, Darcy's law (representing the momentum conservation equation for flow through permeable media), the convection-dispersion equation for concentration (species) and the energy (heat conservation) equation with viscous dissipation for porous media [23]:

$$
\begin{aligned}
& \nabla \cdot V=0 \\
& \nabla P=-\frac{\mu}{K} V \\
& \frac{\partial C}{\partial t}+\frac{1}{\varepsilon_{p}}(V \cdot \nabla) C=D_{C} \nabla^{2} C \\
& \frac{\partial T}{\partial t}+\frac{\lambda}{\varepsilon_{p}}(V \cdot \nabla) T=D_{T} \nabla^{2} T+\frac{\mu}{K\left(\rho C_{P}\right)_{e f f}}\left(u^{2}+v^{2}\right)
\end{aligned}
$$

where $V$ is the velocity vector, $C$ is the concentration, $T$ is the temperature, $\mu$ is the dynamic viscosity as a function of concentration and temperature. $\mathrm{D}_{\mathrm{c}}, \mathrm{D}_{\mathrm{T}}, \mathrm{K}$ and $\varepsilon_{p}$ designate respectively the mass (species) and thermal diffusion coefficient, permeability and porosity of the porous medium, $\lambda$ is the thermal lag coefficient (embodying the ratio of the speed of the thermal front to that of the concentration front) and is mathematically defined as follows: 


$$
\lambda=\frac{\varepsilon_{p} \rho_{f} C_{p_{f}}}{\varepsilon_{p} \rho_{f} C_{p_{f}}+\left(1-\varepsilon_{p}\right) \rho_{s} C_{p s}}
$$

where $\rho$ and $C_{p}$ are the density and specific heat capacity and the indices of $f$ and $s$ refer to the fluid and solid phase, respectively. Due to the heat dissipation, the thermal front always lags behind of the concentration front so $\lambda \leq 1$. It is worth mentioning that the Brinkman term is neglected in the momentum equation since when permeability and porosity are low (as our study), the Darcy-Brinkman law is not valid [24-26] and Darcy law is used instead of it.

The boundary and initial conditions are defined as follows:

$C(x=0, y, t)=C_{1}, \quad T(x=0, y, t)=T_{1}, \quad u(x=0, y, t)=U, \quad v(x=0, y, t)=0$

$$
\begin{aligned}
& \frac{\partial C}{\partial x}\left(x=L_{x}, y, t\right)=0, \quad \frac{\partial T}{\partial x}\left(x=L_{x}, y, t\right)=0 P\left(x=L_{x}, y, t\right)=0, \\
& \frac{\partial T}{\partial y}=0, \frac{\partial C}{\partial y}=0, V=0, \text { at }\left\{\begin{array}{l}
y=0 \\
y=L_{y}
\end{array} \rightarrow \frac{\partial u}{\partial y}=0\right. \\
& T(x, y, t=0)= \begin{cases}T_{1}+f(x, y) & L_{x}<0.06 \mathrm{~m} \\
T_{2}+f(x, y) & L_{x} \geq 0.06 \mathrm{~m}\end{cases} \\
& C(x, y, t=0)= \begin{cases}C_{1}+f(x, y) & L_{x}<0.06 \mathrm{~m} \\
C_{2}+f(x, y) & L_{x} \geq 0.06 \mathrm{~m}\end{cases}
\end{aligned}
$$

where $f(x, y)$ is a random function.

In order to non-dimensionalize the governing equations, the following scaling is used: 


$$
\begin{array}{ccc}
\mathrm{x}^{*}=\frac{\mathrm{x}}{\frac{\mathrm{D}_{\mathrm{c}} \varepsilon_{\mathrm{p}}}{\mathrm{U}}} \quad \mathrm{t}^{*}=\frac{\mathrm{t}}{\frac{\mathrm{D}_{\mathrm{C}} \varepsilon_{\mathrm{p}}{ }^{2}}{\mathrm{U}^{2}}} \quad \mathrm{U}^{*}=\frac{(\mathrm{u} \cdot \mathrm{v})}{\mathrm{U}} & \mathrm{C}^{*}=\frac{\mathrm{C}}{\mathrm{C}_{1}} \\
K^{*}=\frac{K}{\frac{D_{C}{ }^{2} \varepsilon_{p}{ }^{2}}{U^{2}}} \quad P^{*}=\frac{P}{\frac{\mu_{1} U^{2}}{D_{C} \varepsilon_{p}}} \quad \theta^{*}=\frac{\mathrm{T}-\mathrm{T}_{2}}{\mathrm{~T}_{1}-\mathrm{T}_{2}} & \mu^{*}=\frac{\mu}{\mu_{1}}
\end{array}
$$

The dimensionless governing equations can be expressed based on Eqns. (1) - (4) and Eqn. (10):

$$
\frac{\partial u^{*}}{\partial x^{*}}+\frac{\partial v^{*}}{\partial y^{*}}=0
$$

$$
\frac{\partial p^{*}}{\partial x^{*}}=\frac{-\mu^{*} u^{*}}{K^{*}}
$$

$$
\frac{\partial p^{*}}{\partial y^{*}}=\frac{-\mu^{*} v^{*}}{K^{*}}
$$

$$
\frac{\partial C^{*}}{\partial t^{*}}+u^{*} \frac{\partial C^{*}}{\partial x^{*}}+v^{*} \frac{\partial C^{*}}{\partial y^{*}}=\left(\frac{\partial^{2} C^{*}}{\partial x^{* 2}}+\frac{\partial^{2} C^{*}}{\partial y^{* 2}}\right)
$$

$$
\frac{\partial \theta^{*}}{\partial t^{*}}+\lambda\left(u^{*} \frac{\partial \theta^{*}}{\partial x^{*}}+v^{*} \frac{\partial \theta^{*}}{\partial y^{*}}\right)=\operatorname{Le}\left(\frac{\partial^{2} \theta^{*}}{\partial x^{* 2}}+\frac{\partial^{2} \theta^{*}}{\partial y^{* 2}}\right)+B r \cdot L e \frac{\mu^{*}}{K^{*}}\left(u^{*^{2}}+v^{*^{2}}\right)
$$

where $L e=P e_{C} / P e_{T}=D_{T} / D_{C}$ is the Lewis number $\left(P e_{C}=U L_{x} / D_{C}\right.$ and $P e_{T}=U L_{x} / D_{T}$ respectively define the mass and thermal Péclet numbers). $\mathrm{Br}$ is the Brinkman number which embodies the ratio of heat produced by viscous dissipation and heat transported by molecular conduction. Here, the Brinkman number is defined as follows:

$$
B r=\frac{\mu_{1} U^{2}}{k\left(T_{1}-T_{2}\right)}
$$


where $\mathrm{k}$ is the thermal conductivity of fluid.

The boundary and initial conditions can then be written as follows:

$$
\begin{aligned}
& C^{*}\left(x^{*}=0, y^{*}, t^{*}\right)=1, \quad \theta^{*}\left(x^{*}=0, y^{*}, t^{*}\right)=1, \\
& u^{*}\left(x^{*}=0, y^{*}, t^{*}\right)=1, \quad v^{*}\left(x^{*}=0, y^{*}, t^{*}\right)=0 \\
& P^{*}\left(x^{*}=\frac{P e_{C}}{\varepsilon_{P}}, y^{*}, t^{*}\right)=0, \quad \frac{\partial C^{*}}{\partial x^{*}}\left(x^{*}=\frac{P e_{C}}{\varepsilon_{P}}, y^{*}, t^{*}\right)=0, \\
& \frac{\partial \theta^{*}}{\partial x^{*}}\left(x^{*}=\frac{P e_{C}}{\varepsilon_{P}}, y^{*}, t^{*}\right)=0 \\
& a t\left\{\begin{array}{l}
y^{*}=0 \\
y^{*}=\frac{P e_{C}}{A \varepsilon_{P}}
\end{array} \rightarrow \frac{\partial u^{*}}{\partial y^{*}}=0, \frac{\partial \theta^{*}}{\partial y^{*}}=0, \frac{\partial C^{*}}{\partial y^{*}}=0, V^{*}=0\right. \\
& \theta^{*}\left(x^{*}, y^{*}, t^{*}=0\right)= \begin{cases}1+\delta \times \operatorname{rand}(y) & \frac{P e_{C}}{\varepsilon_{p}}<750 \\
0+\delta \times \operatorname{rand}(y) & \frac{P e_{C}}{\varepsilon_{p}} \geq 750\end{cases} \\
& C^{*}\left(x^{*}, y^{*}, t^{*}=0\right)= \begin{cases}1+\delta * \operatorname{rand}(y) * \exp \left(-x^{* 2} / \sigma^{2}\right) & \frac{P e_{C}}{\varepsilon_{p}}<750 \\
0+\delta * \operatorname{rand}(y) * \exp \left(-x^{* 2} / \sigma^{2}\right) & \frac{P e_{C}}{\varepsilon_{p}} \geq 750\end{cases}
\end{aligned}
$$

where, rand is random number between 0 and $1, \delta$ is the magnitude of the disturbance and $\sigma$ can be construed as the penetration of the disturbance from the front.

In non-isothermal miscible displacements, the viscosity depends on temperature and concentration of the fluid. Therefore, to complete the model, the relation between viscosity and concentration and temperature should also be determined. Here, the dimensionless Arrhenius equation is used as the equation of state for viscosity: 


$$
\left.\mu^{*}\left(C^{*}, \theta^{*}\right)=\exp \left(\beta_{C}\left(1-C^{*}\right)+\beta_{T}\left(1-\theta^{*}\right)\right)\right)
$$

where $\beta_{C}=\operatorname{Ln}\left(\mu_{2} / \mu_{1}\right)$ is the mobility ratio and $\mu_{1}$ and $\mu_{2}$ are the displacing and displaced fluid viscosities respectively. $\beta_{T}=\operatorname{Ln}\left(\mu_{T 2} / \mu_{T 1}\right)$ is the thermal mobility ratio and $\mu_{\mathrm{T}_{1}}$ and $\mu_{\mathrm{T}_{2}}$ designate the dynamic viscosities of a single fluid at two different temperatures $\left(T_{1}, T_{2}\right)$.

\section{COMSOL COMPUTATIONAL FLUID DYNAMIC (CFD) SIMULATIONS}

In this study, the classical model of miscible thermal viscous fingering has been examined using the COMSOL multi-physics CFD code (version 5). Here, the miscible thermal viscous fingering displacement in a homogeneous porous medium is successfully simulated. The mapped mesh is applied for discretization of the domain. In our simulations, we assumed that glycerol is the solute and water is the solvent. The problem is solved by employing the twophase Darcy law and heat transfer in porous media models. Two-phase Darcy law couples the steady Darcy flow equation with the time-dependent convection-diffusion equation for the concentration. The model equations are discretized with the finite element method. The resulting system of non-linear equations is solved using the non-linear solver MUMPS [24]. MUMPS (MUltifrontal Massively Parallel sparse direct Solver) is a very efficient parallel sparse direct solver, similar to that employed in ADINA finite element software, and is more robust than conventional iterative solvers which achieves significant active memory reduction and lowers computational costs. For the temporal evolution, the backward Euler method is deployed. It is important to mention that the numerical solution is obtained in a Eulerian system. Further numerical details are available in Bathe [25] and Zimmerman [26] 


\section{COMPUTATIONAL DOMAIN}

The computational domain and boundary conditions for the problem under study are shown in figure 1. At $t^{*}=0$, domain-1 is completely filled with fluid-1 and the rest of the computational domain (domain-2) is filled with fluid-2. At the inlet, the fluid-1 enters with uniform velocity and temperature. Here, a solution with viscosity near to that of water and a 80:20 solution of glycerin and water are considered for fluid-1 and fluid-2, respectively. In other words, the dimensionless groups are calculated based on the properties of these fluids and the defined geometry. The properties of medium are selected near the oil well (oil sand) [27]. Typical values of physical and geometrical parameters used for estimating the dimensionless parameters in the present study are summarized in Table1.

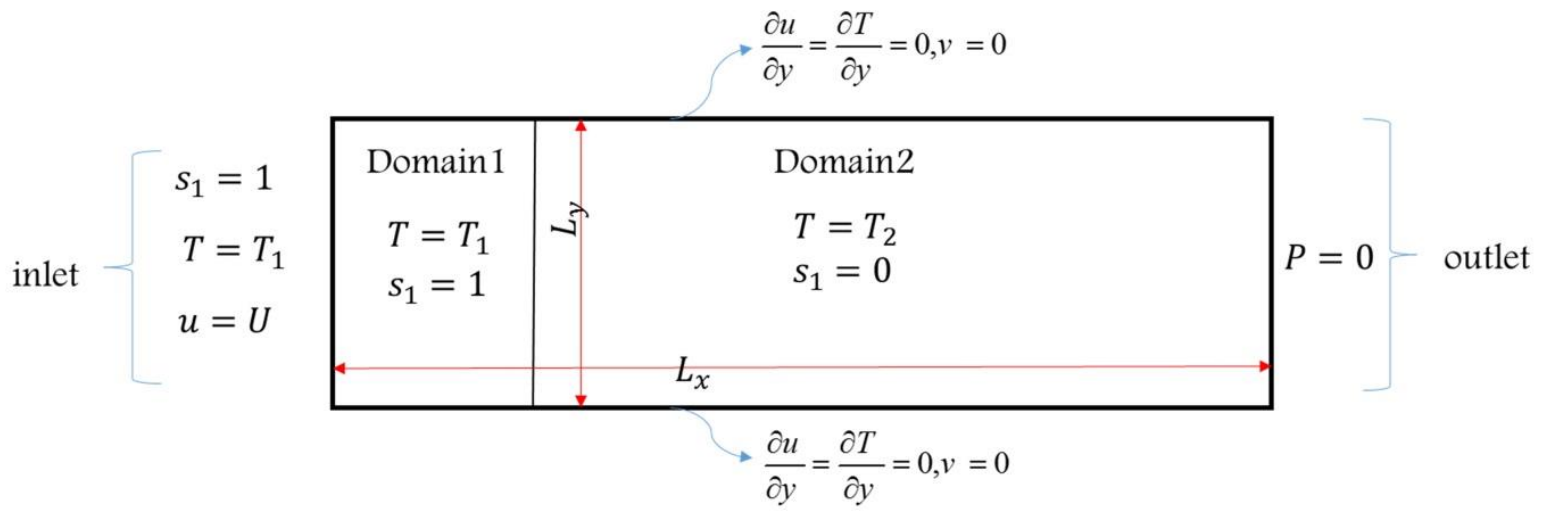

Fig. 1. Schematic of computational domain 


\begin{tabular}{|c|c|c|}
\hline Parameter & Value & Unit \\
\hline$U$ & 0.00177 & $m s^{-1}$ \\
\hline$T_{1}$ & 363 & $K$ \\
\hline$T_{2}$ & 293 & $K$ \\
\hline$\varepsilon_{p}$ & 0.4 & - \\
\hline$K$ & $10^{-12}$ & $\mathrm{~m}^{2}$ \\
\hline$D_{C}$ & $3.54 * 10^{-7}$ & $m^{2} s^{-1}$ \\
\hline$D_{T}$ & $3.54 * 10^{-7}, 7.08 * 10^{-6}, 3.54 * 10^{-5}$ & $m^{2} s^{-1}$ \\
\hline$L_{x}$ & 0.26 & $\mathbf{m}$ \\
\hline$L_{y}$ & 0.08 & $\mathbf{m}$ \\
\hline$B r$ & $\frac{\mu_{1} U^{2}}{k\left(T_{2}-T_{1}\right)}=\frac{0.001 *(0.00177)^{2}}{0.44 * 70}=10^{-10}$ & - \\
\hline$P e_{C}$ & $\frac{U L_{x}}{D_{C}}=\frac{.00177 * 0.26}{3.54 * 10^{-7}}=1300$ & - \\
\hline Le & $\frac{P e_{C}}{P e_{T}}=\frac{D_{T}}{D_{C}}=1,20,100$ & - \\
\hline$A$ & $\frac{L_{x}}{L_{y}}=\frac{0.26}{0.08}=3.25$ & - \\
\hline
\end{tabular}

It is worth mentioned that considering values of Table 1, could the viscous dissipation be ignored? If we return to equation 4, the RHS of Eq.4 contains conduction and viscous dissipation terms. The order of magnitude of these terms are $O\left(\frac{k \Delta T}{\rho C \frac{D_{C}{ }^{2} \varepsilon_{p}{ }^{2}}{U^{2}}}\right)$ and $O\left(\frac{\mu U^{2}}{K \rho C_{P}}\right)$ , respectively. Therefore the viscous dissipation is ignored if $\frac{\mu U^{2}}{K \rho C_{P}}=\frac{k \Delta T}{\rho C \frac{D_{C}{ }^{2} \varepsilon_{p}{ }^{2}}{U^{2}}}$ or in 
other words, $\frac{\frac{\mu U^{2}}{K \rho C_{P}}}{\frac{k \Delta T}{\rho C_{p} L^{2}}}=1 \Rightarrow \frac{\frac{\mu U^{2}}{k \Delta T}}{\frac{\frac{K}{D_{C}{ }^{2} \varepsilon_{p}{ }^{2}}}{U^{2}}}=1 \Rightarrow \frac{B r}{K^{*}}=1$. According to table 1 , we have, $\mathrm{Br} / \mathrm{K}^{*}=100$, therefore, for this situation, the viscous dissipation could not be ignored.

\section{LINEAR STABILITY ANALYSIS}

Prior to present the results of nonlinear simulations, we will first examine the linear stability of thermal viscous fingering. This leads to an initial view of the effect of the main parameters on the flow instability. For this purpose, as same as many studies in this field $[7-11,18]$, a Lagrangian reference frame is chosen that moves with the superficial velocity. In addition of convenience, this is useful to more detailed analysis (By creating the possibility of examining the effect of Brinkman number). Then, a base state solution is considered for the governing equations and a set of small disturbances is added to them as follows:

$$
\begin{aligned}
& u^{*}(x, y, t)=u_{0}^{*}(x, t)+u^{\prime *}(x, y, t) \\
& v^{*}(x, y, t)=v_{0}(x, t)+v^{\prime *}(x, y, t) \\
& C^{*}(x, y, t)=C_{0}{ }^{*}(x, t)+C^{\prime *}(x, y, t) \\
& \mu^{*}(x, y, t)=\mu_{0}^{*}(x, t)+\mu^{\prime *}(x, y, t) \\
& \theta^{*}(x, y, t)=\theta_{0}^{*}(x, t)+\theta^{\prime *}(x, y, t) \\
& p^{*}(x, y, t)=p_{0}{ }_{0}(x, t)+p^{\prime^{*}}(x, y, t)
\end{aligned}
$$

In the above equations, the primed quantities represent small disturbances and the base states are denoted by zero subscript.

The linearized equations are:

$$
\frac{\partial u^{\prime *}}{\partial x^{*}}+\frac{\partial v^{\prime *}}{\partial y^{*}}=0
$$




$$
\begin{aligned}
& \frac{\partial p^{\prime *}}{\partial x^{*}}=-\frac{\mu_{0}^{*} u^{\prime *}}{K^{*}}-\frac{\mu^{\prime *}}{K^{*}} \\
& \frac{\partial p^{\prime *}}{\partial y^{*}}=-\frac{\mu_{0}^{*} v^{\prime *}}{K^{*}} \\
& \frac{\partial C^{\prime *}}{\partial t^{*}}+u^{\prime^{*}} \frac{d C_{0}^{*}}{d x^{*}}=\frac{\partial^{2} C^{\prime *}}{\partial x^{* 2}}+\frac{\partial^{2} C^{\prime *}}{\partial y^{* 2}} \\
& \frac{\partial \theta^{\prime *}}{\partial t^{*}}+\lambda u^{\prime *} \frac{\partial \theta_{0}^{*}}{\partial x^{*}}+(\lambda-1) \frac{\partial \theta^{\prime *}}{\partial x^{*}}=L e\left(\frac{\partial^{2} \theta^{\prime *}}{\partial x^{* 2}}+\frac{\partial^{2} \theta^{\prime *}}{\partial y^{* 2}}\right)+\frac{B r \cdot L e}{K^{*}}\left(\mu^{\prime *}+\mu_{0}^{*}\left(2 u^{\prime *}\right)\right) \\
& \mu^{\prime *}=-\mu_{0}^{*}\left(\beta_{C} C^{\prime *}+\beta_{T} \theta^{\prime *}\right)
\end{aligned}
$$

The equations (24-26) can be combined as:

$$
\left(\frac{\partial^{2} u^{\prime *}}{\partial x^{* 2}}+\frac{\partial^{2} u^{\prime *}}{\partial y^{* 2}}\right)+\left(\frac{\partial^{2} \mu^{\prime *}}{\mu_{0}^{*} \partial y^{* 2}}\right)+\left(\frac{\partial u^{\prime *}}{\partial x^{*}}\right)\left(\frac{\partial \mu_{0}^{*}}{\mu_{0}^{*} \partial x^{*}}\right)=0
$$

Thus, the linearized equations can be expressed in terms of only the concentration disturbance $C^{\prime *}$, the temperature disturbance $\theta^{\prime *}$ and the streamwise velocity disturbance $u^{\prime *}$.

Considering the base state solution of the governing equations, the base state concentration, temperature and viscosity are obtained as follows:

$$
\begin{aligned}
& C_{0}^{*}=\frac{1}{2}\left(1-\text { erf }\left(\frac{x^{*}}{2 \sqrt{t^{*}}}\right)\right) \\
& \theta_{0}^{*}=\frac{1}{2}\left(1-\operatorname{erf}\left(\frac{x^{*}-(\lambda-1) t^{*}}{2 \sqrt{\text { Let }^{*}}}\right)\right) \\
& \left.\mu_{0}^{*}=\exp \left(\beta_{C}\left(1-C_{0}^{*}\right)+\beta_{T}\left(1-\theta_{0}^{*}\right)\right)\right)
\end{aligned}
$$


Using the quasi-steady state approximation, the disturbances are expanded in terms of Fourier components as:

$$
\left(C^{\prime *}, u^{\prime *}, \theta^{\prime *}\right)=(C, U, \theta)(x *) e^{\sigma\left(t_{0}\right) t^{*}} e^{i k y^{*}}
$$

Where ${ }_{k}$, and $\sigma\left(t_{0}\right)$ are the wave number of the disturbances in the direction and growth rate of the disturbance, respectively.

Substituting equation (34) into linearized equations, we have:

$$
\left.\begin{array}{l}
\left.\frac{d^{2}}{d x^{* 2}}-k^{\prime 2}-\left(\beta_{C} \frac{d C_{0}^{*}}{d x^{*}}+\beta_{T} \frac{d \theta_{0}^{*}}{d x^{*}}\right) \frac{d}{d x^{*}}\right) U+k^{\prime 2}\left(\beta_{C} C+\beta_{T} \theta\right)=0 \\
\left(\sigma\left(t_{0}\right)-\frac{d^{2}}{d x^{* 2}}+k^{\prime 2}\right) C=-U \frac{d C_{0}^{*}}{d x^{*}} \\
\left(\sigma\left(t_{0}\right)+(\lambda-1) \frac{d}{d x^{*}}+\operatorname{Le}\left(-\frac{d^{2}}{d x^{* 2}}+k^{\prime 2}\right)\right) \theta=-\lambda U \frac{d \theta_{0}^{*}}{d x^{*}}+\frac{B r \cdot L e}{K^{*}} \mu_{0}^{*}\left(-\left(\beta_{C} C+\beta_{T} \theta\right)+2 U\right)
\end{array}\right)
$$

The above eigenvalue differential system is solved numerically using the sixth order shooting method. The largest eigenvalues in the computational domain is only reported. We chose this domain to be sufficiently wide to capture all the eigen-solutions. In order to compare and check the validity of the present model, the comparisons between the predictions of our analysis with $B r=0$ and the results of Islam and Azaiez [18] are shown in Figure 2. In this figure, the results obtained from our simulations are shown with line and the results of Islam and Azaiez [18] are represented as circles for $\beta_{T}=2$ and square for $\beta_{T}=1$ where $\beta_{C}=1, \lambda=1$, $L e=20, P e_{C}=500$ and $t_{0}=10$. 


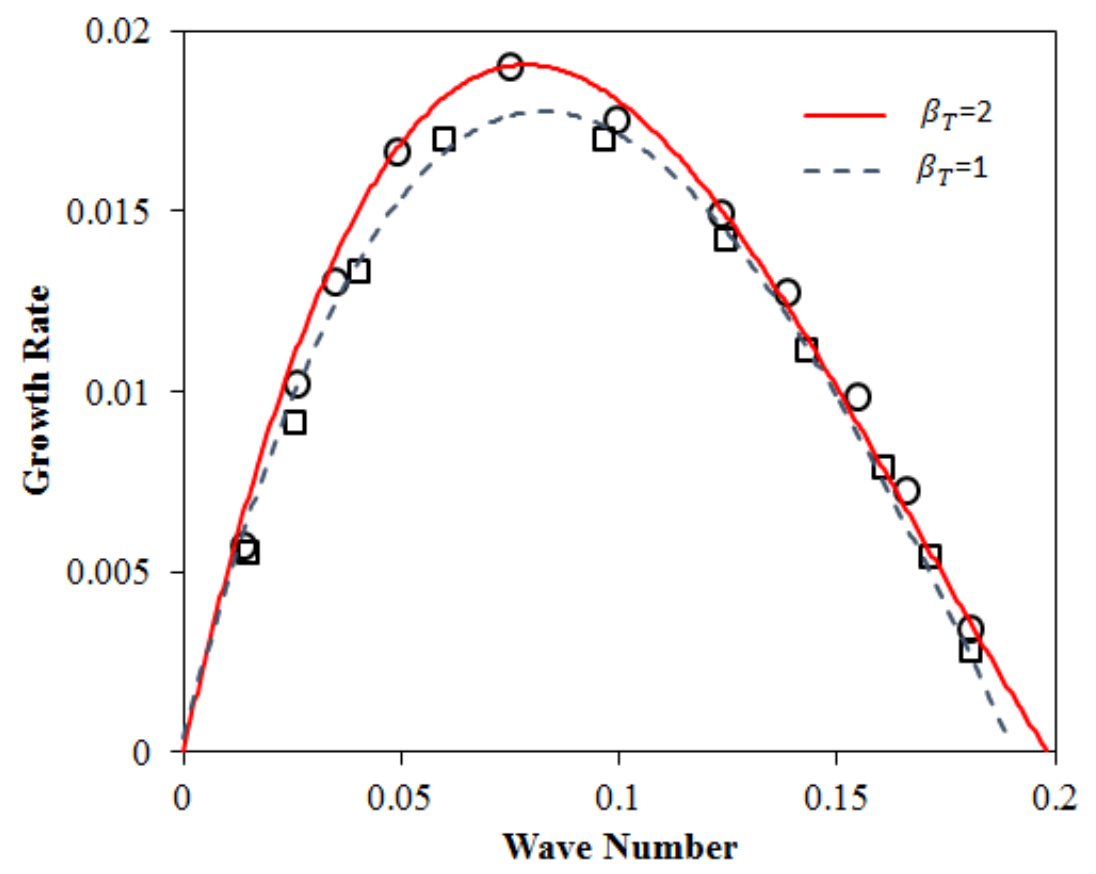

Fig. 2 Instability characteristic for $\beta_{C}=1, \lambda=1, L e=20, P e_{C}=500$ and $t_{0}=10$ : comparison between the present results and Islam and Azaiez [18].

Figure 3 shows the variation of the growth rate of disturbance as a function of the thermal lag coefficient $(\lambda)$ and the wave numbers for $\beta_{C}=3, \beta_{T}=2, L e=1, B r=10^{-10}$ and $t^{*}{ }_{0}=0.1$. It can be seen that the flow becomes more stable by decreasing $\lambda$. This is attributable to a decrease in the destabilizing effect of the thermal front on the concentration front when their distance from each other is increased. 


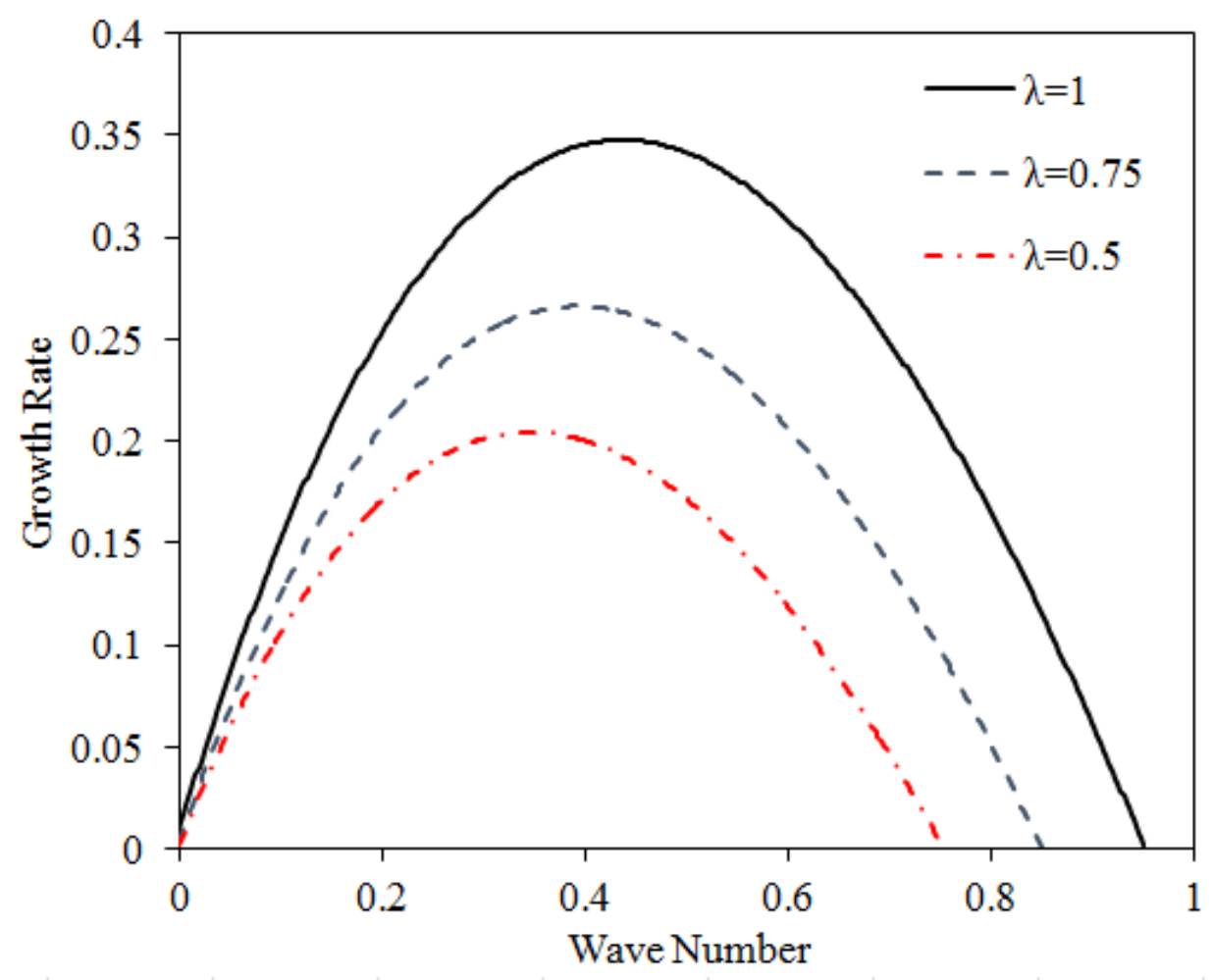

Fig.3 Instability characteristic at different values of $\lambda$ for $\beta_{C}=3, \beta_{T}=2, L e=1$, $B r=10^{-10}$ and $t^{*}{ }_{0}=0.1$.

The variation of the growth rate of disturbance $\sigma$ for $\beta_{C}=3, \beta_{T}=2, \lambda=0.75, B r=10^{-10}$ and $t^{*}{ }_{0}=0.1$ versus the wave number for different Lewis number $(L e)$ is shown in Figure 4. According to the figure, the flow is more stable by increasing the Lewis number. The effect of the Brinkman number on the growth rate and wave number at $\beta_{C}=3, \beta_{T}=2, \lambda=0.75, L e=1$ and $t^{*}{ }_{0}=0.1$ is shown in Figure 5. It can be seen that the Brinkman number has a stabilizing effect on the flow. However due to neglection in higher order terms, this method could not completely show the effect of this parameter and nonlinear simulation will therefore be more efficient. 


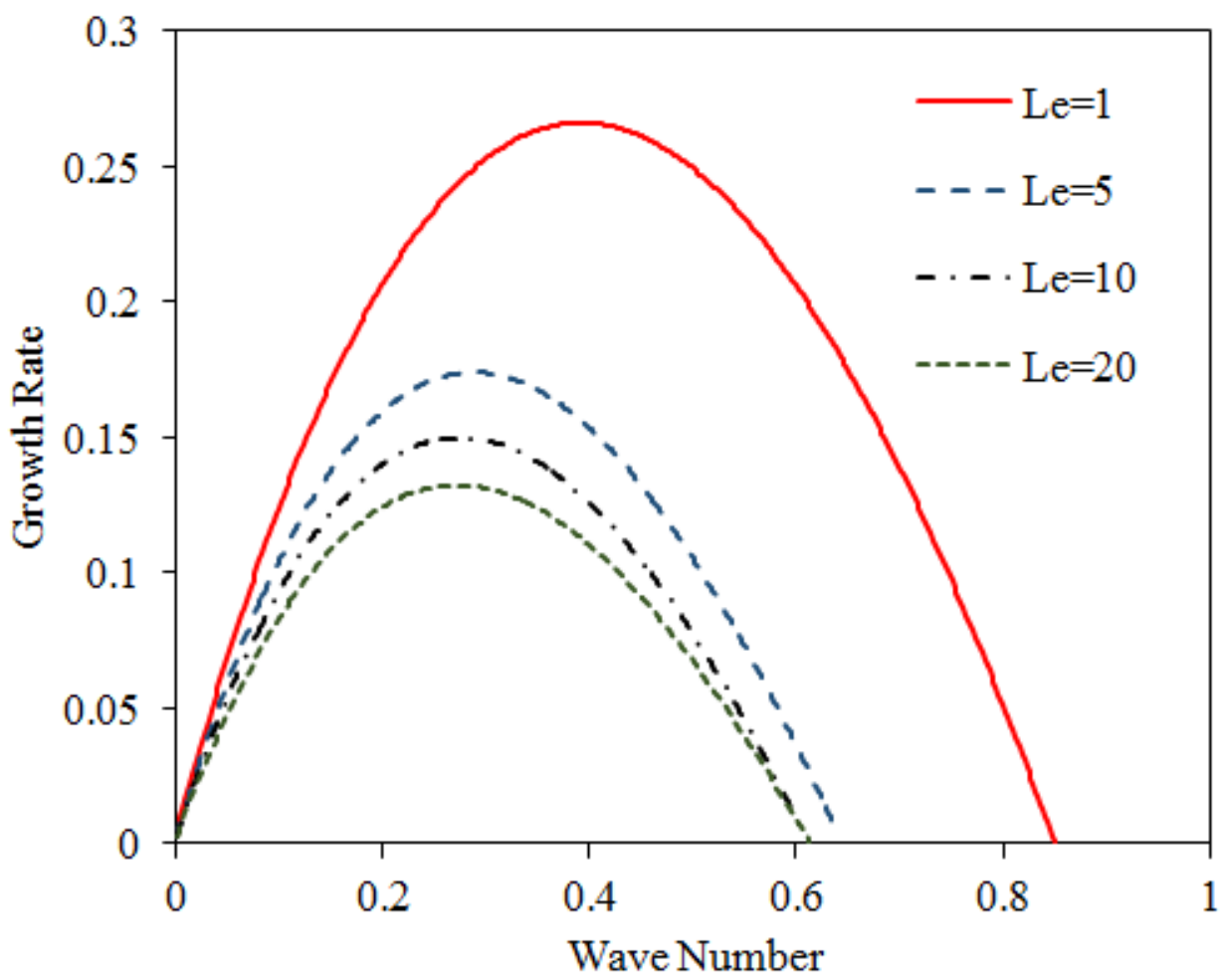

Fig.4 Instability characteristic at different values of $L e$ for $\beta_{C}=3, \beta_{T}=2$, $\lambda=0.75, B r=10^{-10}$ and $t^{*}{ }_{o}=0.1$.

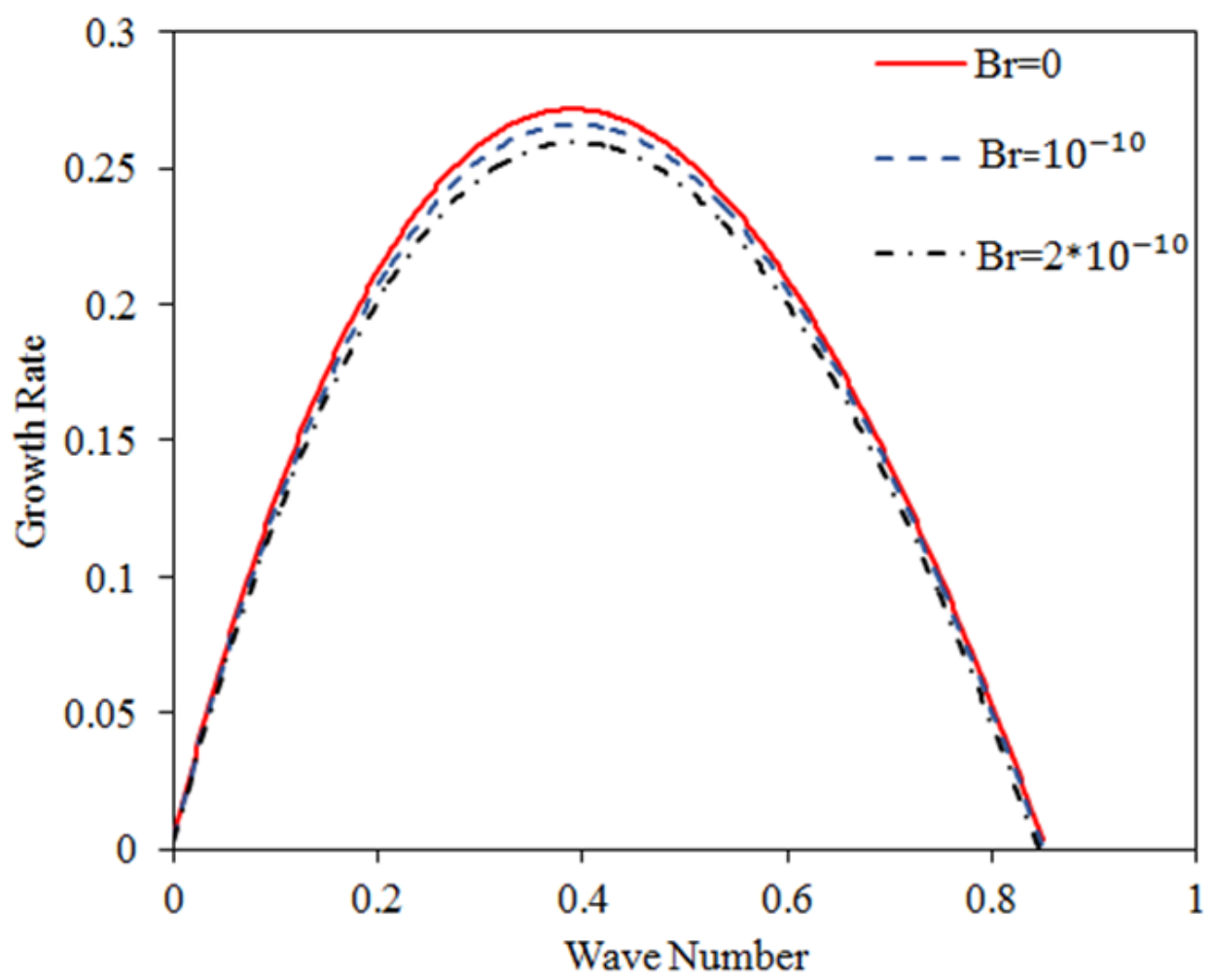


Fig.5 Instability characteristic at different values of $B r$ for $\beta_{C}=3, \beta_{T}=2, \lambda=0.75, L e=1$ and $t^{*}{ }_{0}=0.1$.

\section{NUMERICAL RESULTS AND DISCUSSION}

In this section, the results of the nonlinear simulations of thermal viscous fingering are presented and the effect of viscous dissipation on this instability is investigated. The results include concentration and temperature contours, transversely averaged profiles, mixing length, sweep efficiency. Furthermore, the effects of Lewis number and the thermal lag coefficient on this instability is studied in detail. Unless otherwise mentioned, as presented in Table 1, the flows are analyzed for $P e_{C}=1300, \beta_{T}=2, \beta_{C}=3$ and $\mathrm{Br}=10^{-10}$

\section{a. Grid Study and Verification}

To obtain a grid-independent solution, we consider various structured grids. Table 2 lists the grids utilized and the corresponding grid numbers. Due to the random nature of viscous fingering instability, the sweep efficiency is used to determine the level of dependency of numerical solution to grid numbers. This parameter is an average result of CFD simulation that is obtained from all the computational grids. The sweep efficiency of different meshes at $L e=1$ and $\lambda=1$ are shown in Figure 6. It is evident that sweep efficiency is approximately equal for grids M-3, M-4 and M-5. To ensure that the obtained solution is grid-independent and in order to avoid heavy computational cost, we used the grids M-4 for all subsequent CFD simulations since these will guarantee the necessary resolution of the flow field and adequate accuracy.

Table 2. Characteristics of the computational meshes for grid study

\begin{tabular}{cccc}
\hline Grid & $\begin{array}{c}\text { Number of cells for } \\
\text { domain-1 }\end{array}$ & $\begin{array}{c}\text { Number of cells for } \\
\text { domain-2 }\end{array}$ & $\begin{array}{c}\text { Total number of } \\
\text { cells }\end{array}$ \\
\hline M-1 & 6600 & 22650 & 29250 \\
\hline M-2 & 14850 & 51075 & 65925 \\
\hline M-3 & 26400 & 90600 & 117000 \\
\hline
\end{tabular}




\begin{tabular}{cccc}
\hline M-4 & 59400 & 203850 & 263250 \\
\hline M-5 & 105600 & 362400 & 468000 \\
\hline
\end{tabular}

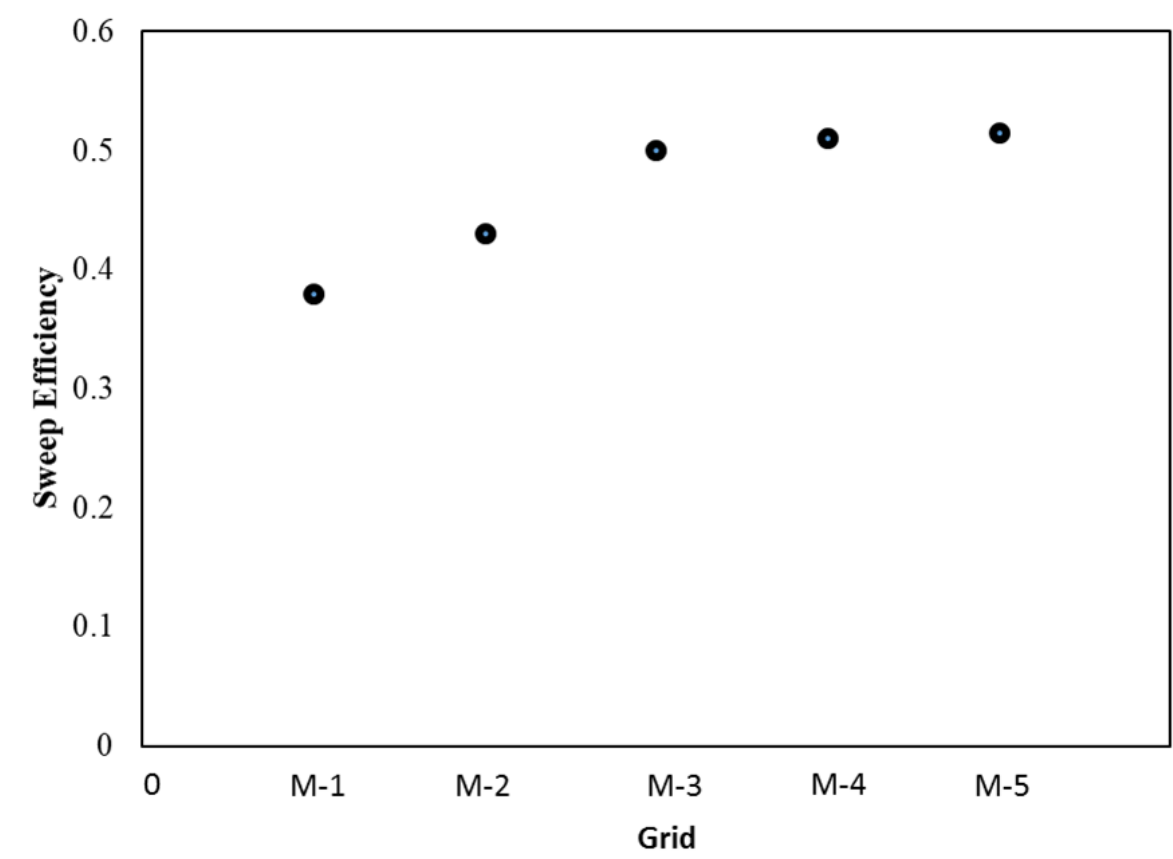

Fig. 6. The sweep efficiency obtained from different grids at $L e=1$ and $\lambda=1$

Prior to analyzing the effects of different parameters on the thermal fingering instability, it is necessary to verify the results. For this purpose, a comparison between numerical simulation and linear growth rate analysis is done to validate our numerical code. Here, as in the earlier study of Tan and Homsy [28], the concentration perturbation are considered as a single mode in the transverse direction and $0.1 \%$ in magnitude and the average growth rate of the discrete mode is computed at an early time and compared with linear growth rate analysis results. Figure 7 shows this comparison. Inspection of the figure reveals that the results obtained from the COMSOL CFD simulations demonstrate very close correlation with those predicted by linear stability analysis over a range of wave numbers. Confidence in the COMSOL computations is therefore justifiably high. 


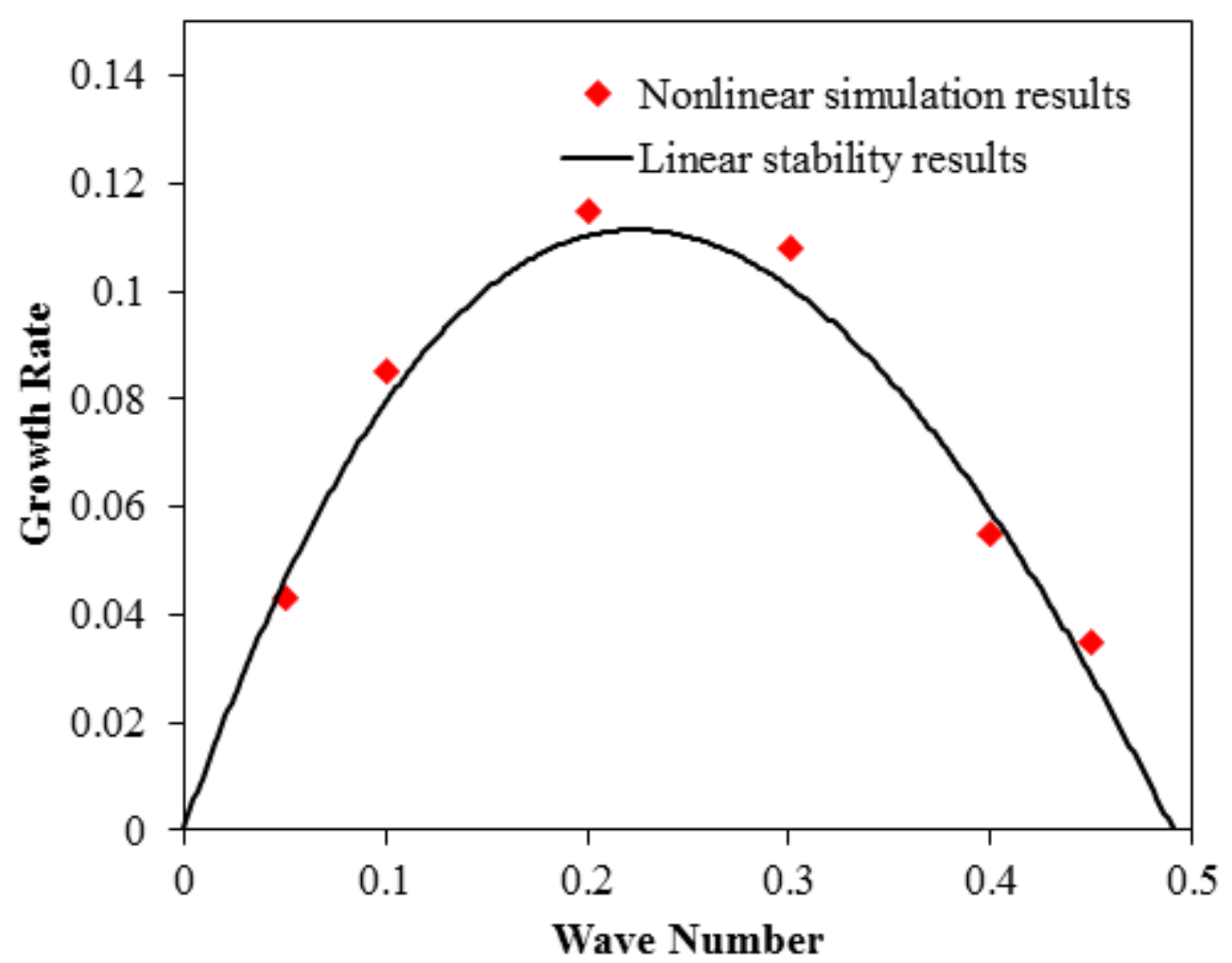

Fig. 7 Comparison of the initial growth rate between the full simulation and the linear stability analysis $\left(\lambda=0.75, L e=1\right.$ and $\left.t_{0}^{*}=10\right)$

\section{b. Concentration and temperature contours}

We first study the role of viscous dissipation on the growth of instability. For this purpose, the obtained results of nonlinear simulation with viscous dissipation included $(\mathrm{Br} \neq 0)$ are compared with the equivalent flow without this term $(B r=0)$. Figure 8 shows the concentration and temperature contours for both dissipative and non-dissipative cases with $\mathrm{Le}=1$ and $\lambda=1$. It is noted that in the absence of viscous dissipation, the fingers grow faster and reach the end of the domain. However, in the case of with viscous dissipation, the fingers grow more regularly but distinctly more slowly. This is due to the fact that viscous dissipation increases the temperature of the flow and hence its viscosity (which is a function of temperature) decreases. Since the displaced fluid has a higher viscosity than the displacing one $\left(\mu_{1}<\mu_{2}\right)$, it is faced with stronger viscous hydrodynamic resistance than the displacing fluid. Therefore, the ratio of viscosity and the intensity of viscous fingering instability are decreased. The delay in reaching the displacing fluid to the end of the path also encourages the entry of a supplementary volume of displacing fluid to the area. As a result, more displaced fluid is forced 
to migrate out of the domain. From comparison between contours, it can be concluded that decreases the length of fingers. It can also be seen that the concentration and temperature fronts are similar and bunched together at $\lambda=1$. In this condition, there is no heat transfer between the solid and the fluid phases and the convection-diffusion and energy equations act similar to each other at $\lambda=1$ and Le $=1$. For the case of $B r=0$, the double coalescence mechanism is identified by a circle in the frame at $t^{*}=515$. In this mechanism, two fingers slowly bend to their middle finger and merge into its body thereby creating a wider finger.
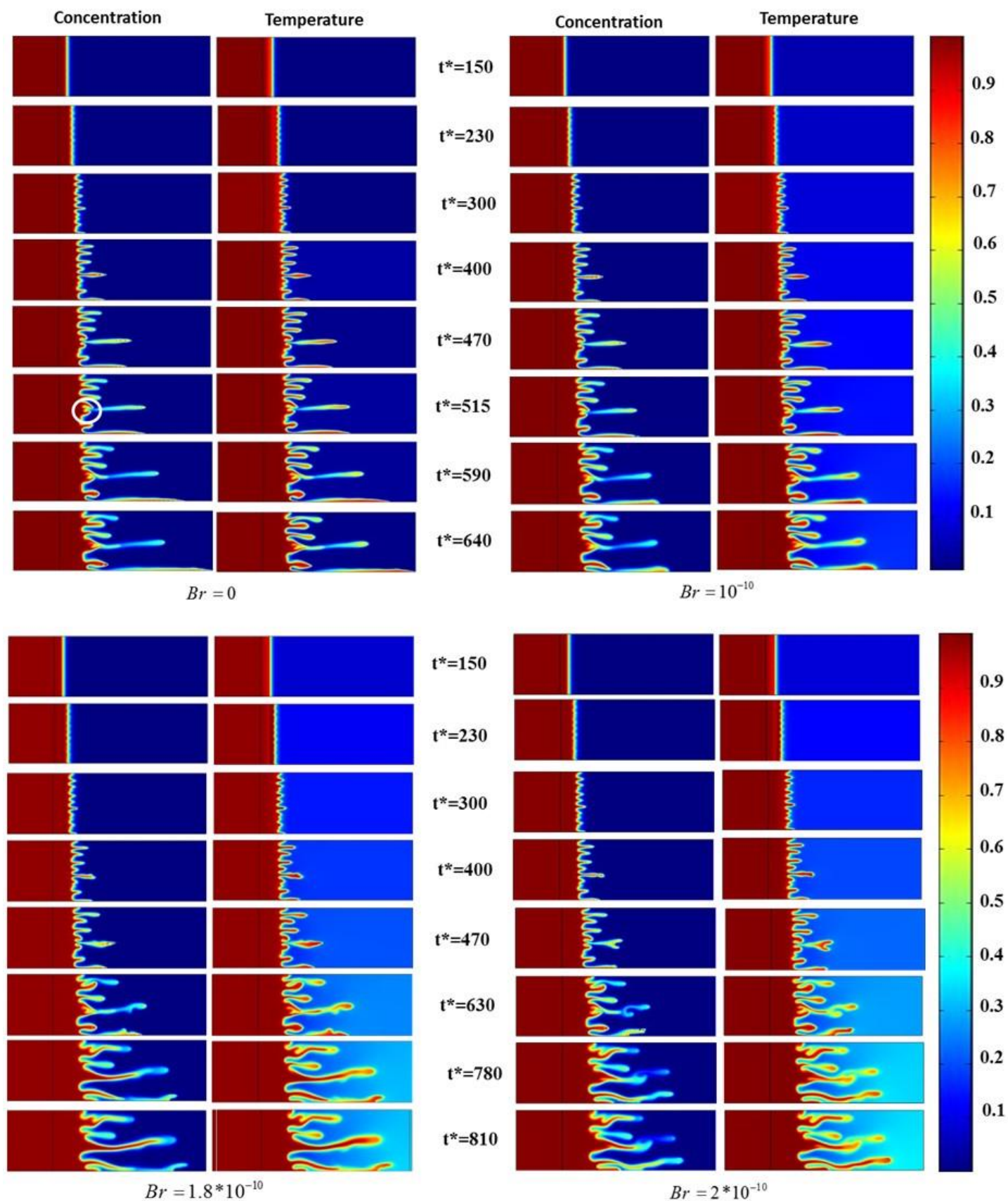

$t^{*}=630$
$t^{*}=780$
$t^{*}=810$

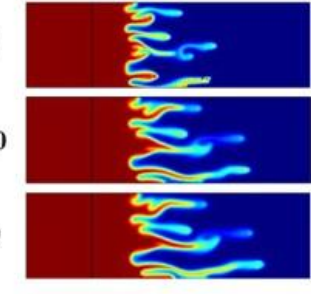

$B r=2 * 10^{-10}$

Fig. 8 The effect of viscous dissipation on concentration and temperature 
Figure 9 depicts the concentration and temperature contours for Le $=1$ and two values of the thermal lag coefficient, $\lambda=0.5$ and $\lambda=0.75$. According to the figure, the thermal front lags behind the concentration front for $\lambda<1$. Indeed, decreasing $\lambda$ leads to an increase in the rate of heat dissipation. Therefore, the velocity of thermal front decreases $\left(U_{T}=\lambda U\right)$ and it lags behind the concentration front. The destabilizing effect of the thermal front on the concentration front is decreased and the flow becomes more stable by decreasing $\lambda$. The double coalescence mechanism is identified by a circle in the frame at $t^{*}=550$ and the spreading mechanism is observed in the frame at $t^{*}=700$. In this mechanism, the finger morphs from its horizontal form and becomes wider.
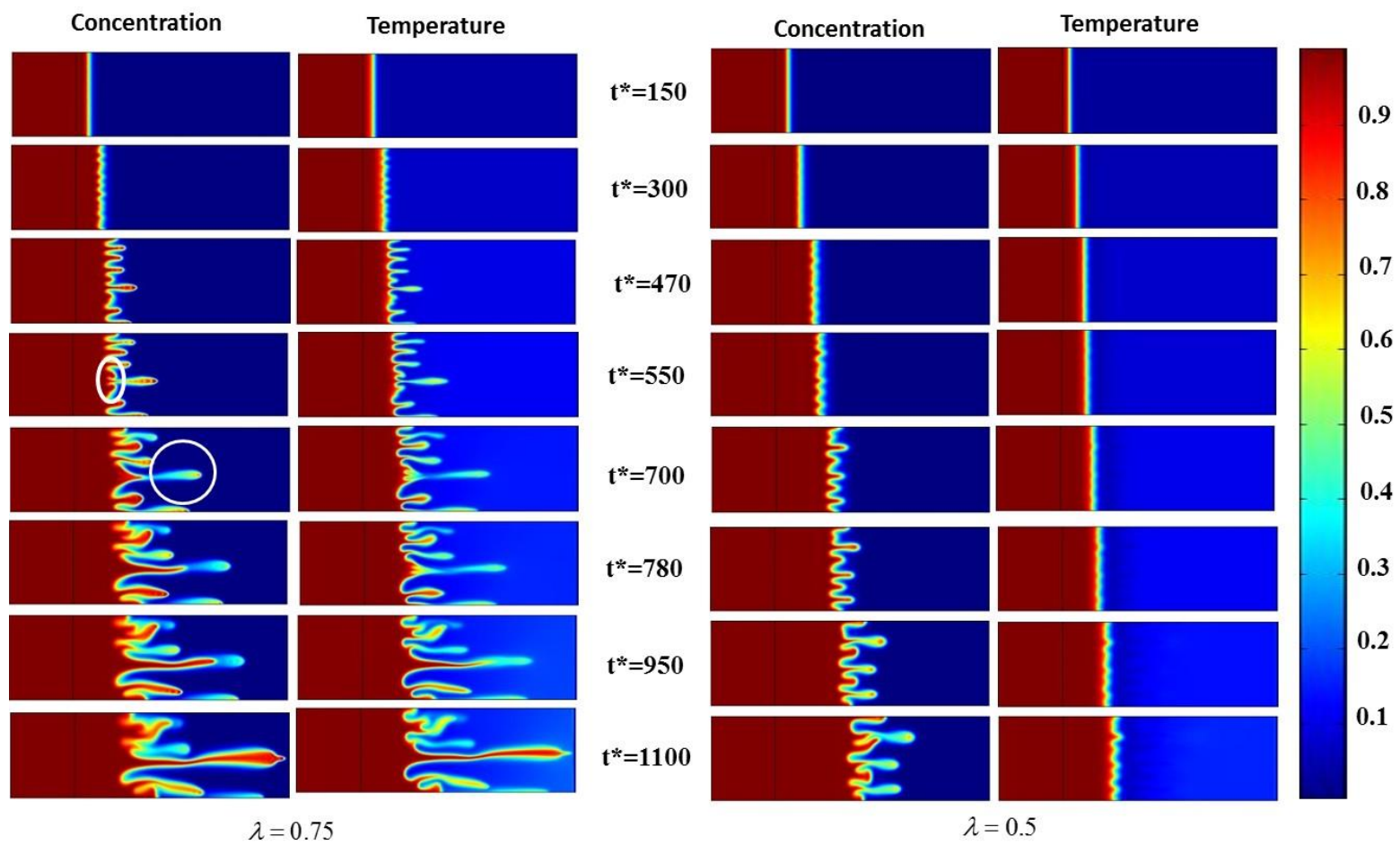

Fig. 9 The effect of thermal lag coefficient on concentration and temperature contours

$$
\text { ( } \left.L e=1 \text { and } B r=10^{-10}\right)
$$

The effect of Lewis number on the concentration and temperature contours is shown in Figure 10. The Lewis number expresses the ratio of heat diffusion to the molecular diffusion. Via comparing temperature contours, it is observed that by increasing the Lewis number, the thermal front diffuses with a weakly wavy front and no major fingers are featured. A comparison between the concentration contours at the same time shows that the intensity of instability decreases by increasing the Lewis number. Indeed, it can be concluded that by 
increasing the Lewis number, the destabilizing effect of the thermal front on the concentration front is decreased and the level of instability is reduced. A specific type of the tip-splitting mechanism is observed in the frame at $t^{*}=1000$. Herein the tip of the finger bifurcates whereas the penetrating adjacent finger in its body (the coalescence mechanism at $t^{*}=1100$ ) re-vitalizes this finger and prevents the subsequent creation of two branching-fingers at this tip. Additionally, the spreading mechanism is identified by an ellipse in the frame at $t^{*}=1450$

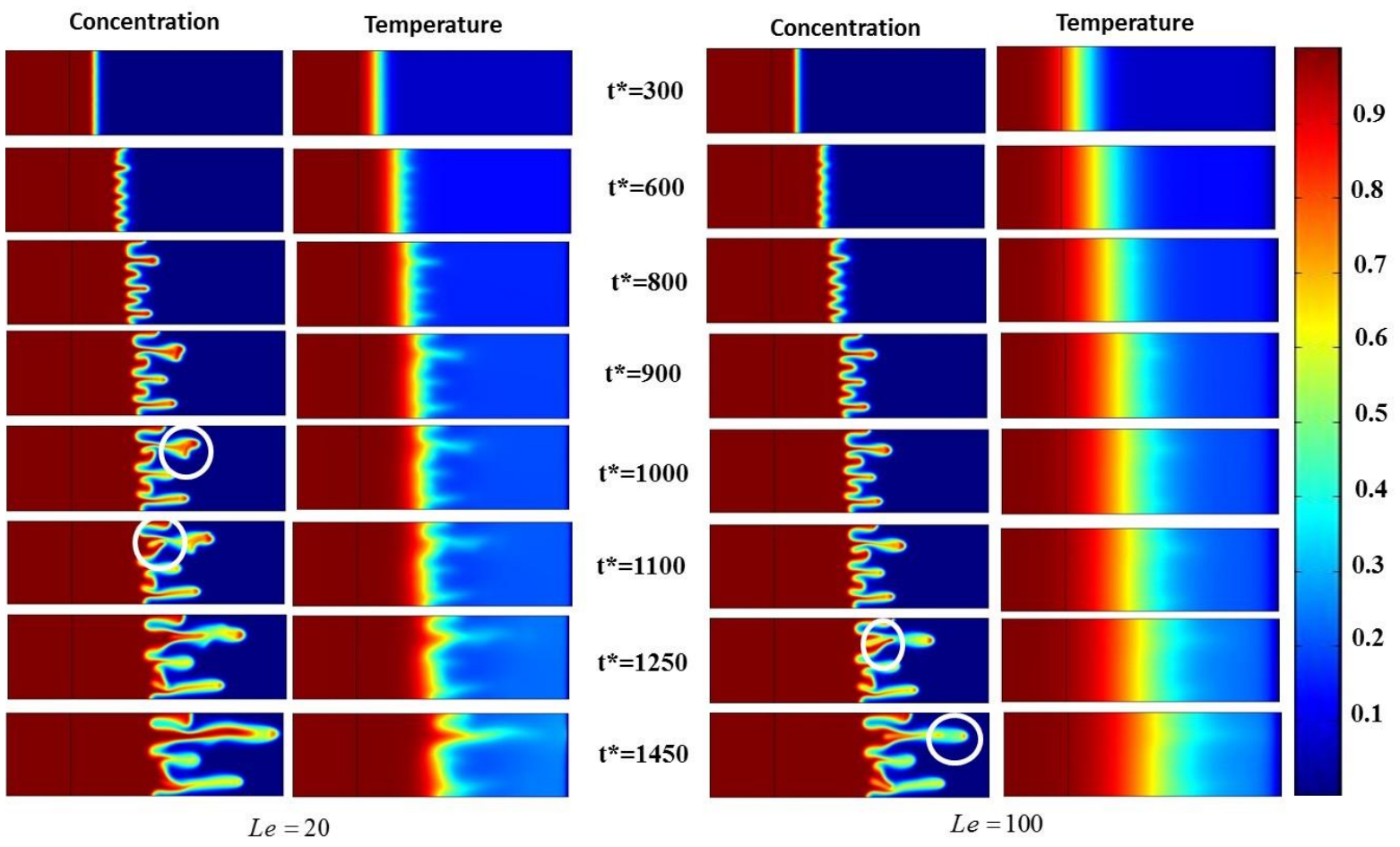

Fig. 10 The effect of Lewis number on concentration and temperature contours ( $\lambda=0.75$ and

$$
B r=10^{-10} \text { ) }
$$

\section{c. Transversely Averaged Profiles}

Inspecting the transversely averaged profile is a classical tool employed for measuring the intensity of viscous fingering instability in experimental and theoretical studies. Indeed, the study of results obtained from measurement of different parameters in one spatial dimension is a suitable approach for understanding the flow behavior in different media. Transversely and longitudinally averaged one-dimensional concentration profiles have been presented by Zimmerman and Homsy [29] to investigate the fingering instability in porous media with anisotropic dispersion. In the present study, the transversely averaged concentration and temperature profiles are investigated for some scenarios both with and without viscous 
dissipation. These parameters as a function of time and longitudinal position can be defined as follows:

$$
\begin{aligned}
& \bar{C}\left(x^{*}, t^{*}\right)=\frac{A \varepsilon_{p}}{P e_{C}} \int_{0}^{\frac{P e_{C}}{\varepsilon_{p} A}} C^{*}\left(x^{*}, y^{*}, t^{*}\right) d y^{*} \\
& \bar{\theta}\left(x^{*}, t^{*}\right)=\frac{A \varepsilon_{p}}{P e_{C}} \int_{0}^{\frac{P e_{C}}{\varepsilon_{p} A}} \theta^{*}\left(x^{*}, y^{*}, t^{*}\right) d y^{*}
\end{aligned}
$$

The transversely averaged concentration profiles at $B r=10^{-10}$ and $L e=\lambda=1$ for different times are shown in figure 11. According to the figure, at the initial times, the diagrams exhibit no oscillations. With time elapse the fingers grow upwards and some peaks are created in the profiles. An increasing number of peaks in the transversely average profiles implies that the flow becomes more unstable.

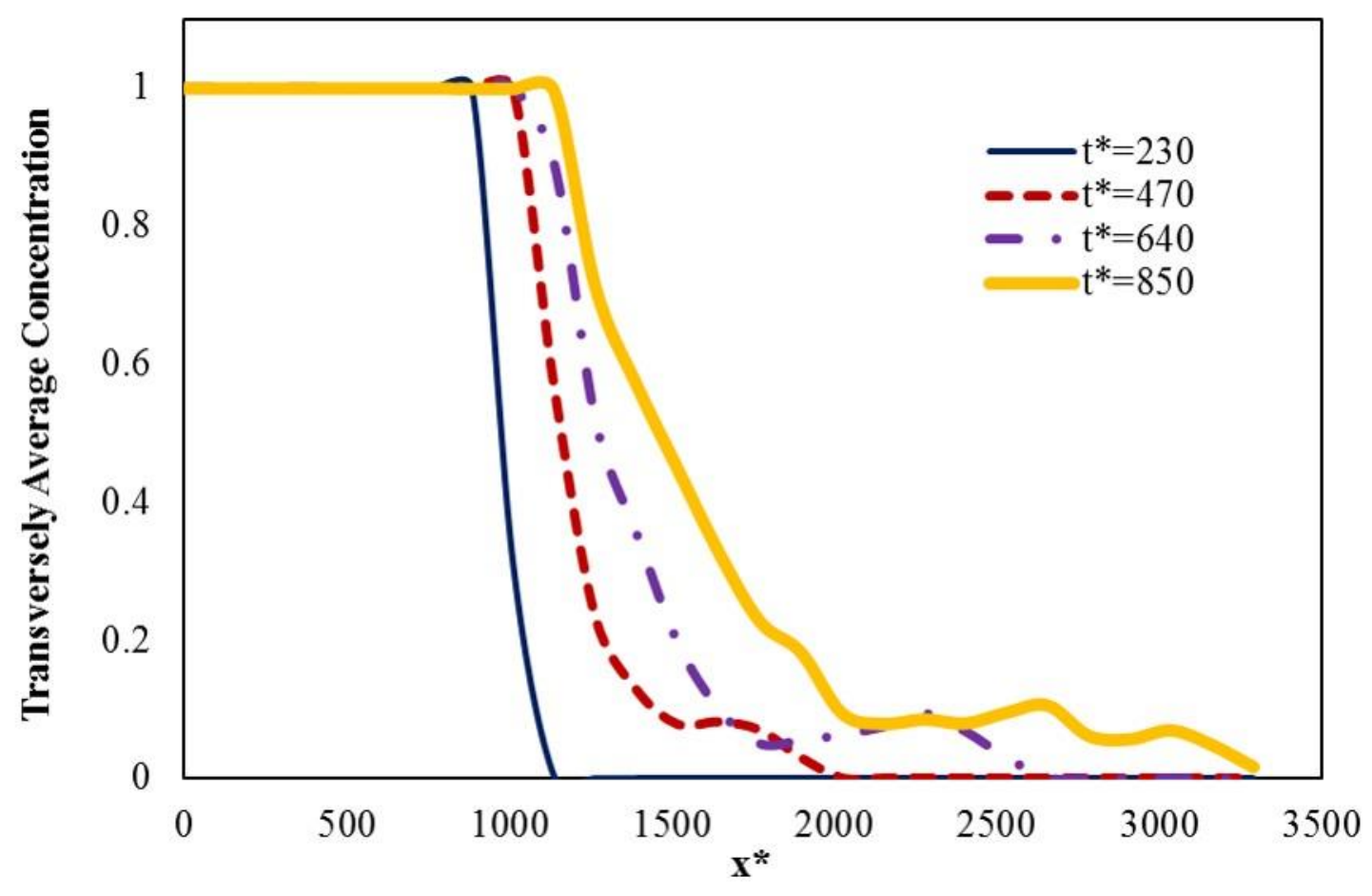

Fig11 Transversely averaged concentration profiles for $B r=10^{-10}$ and $L e=\lambda=1$.

Figure 12 shows the transversely averaged temperature profiles at different time instants. As seen in the figure, the temperature is enhanced with the passage of time. This increase in 
temperature is higher in the displaced fluid since it possesses a higher viscosity and the viscous dissipation is also higher. Distortion of curves also increases as the time increases resulting in increasing instability in the flow.

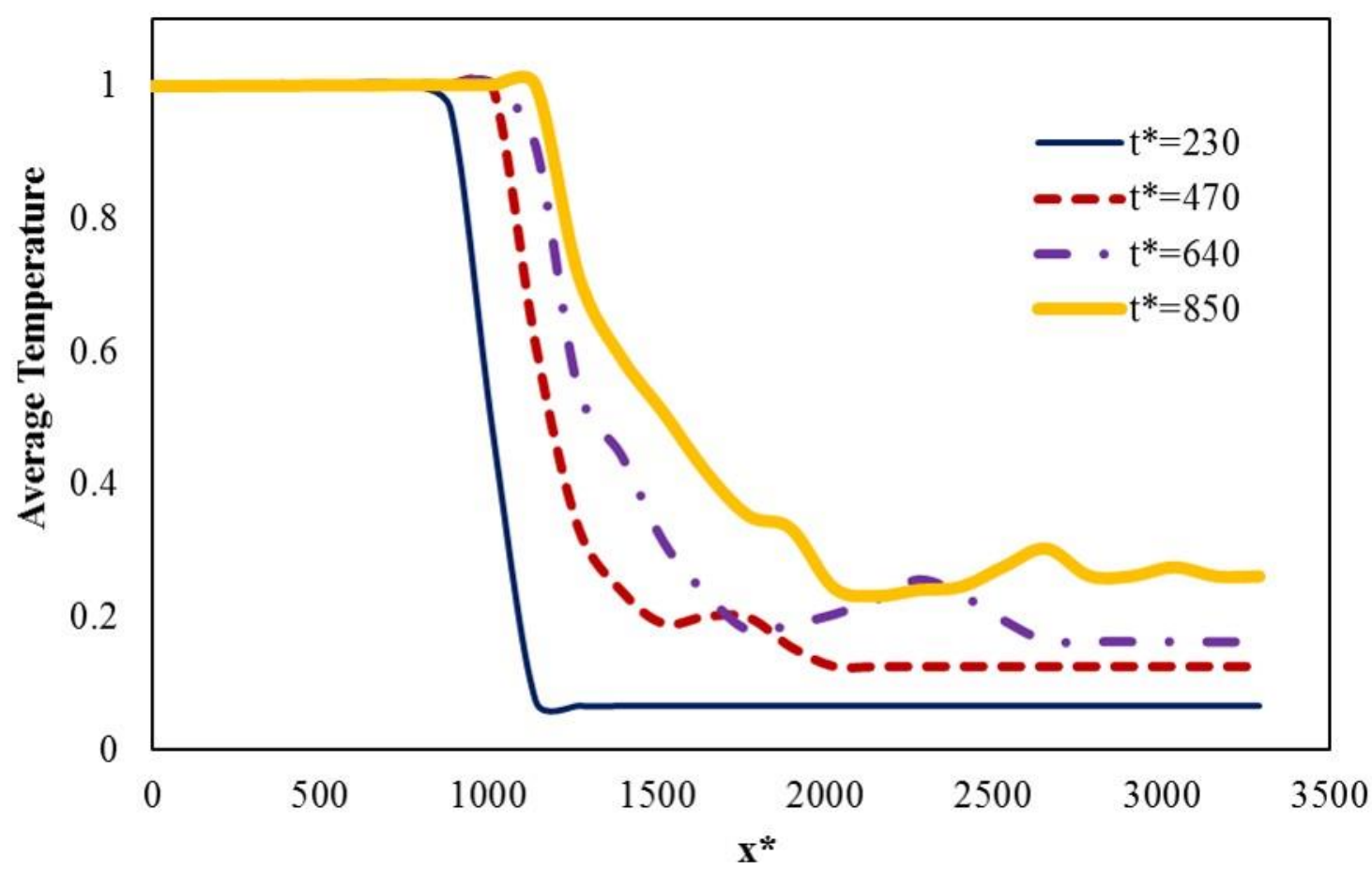

Fig. 12 Transversely averaged temperature profiles for $B r=10^{-10}$ and $L e=\lambda=1$.

The transversely averaged concentration and temperature profiles at $\mathrm{Br}=0, \mathrm{Br}=10^{-10}$ and $t^{*}=640$ are shown in Figure 13. As mentioned before and visualized in the concentration and temperature contours, viscous dissipation increases the temperature, decreases the viscosity ratio and reduces the level of hydrodynamic instability. The same deduction may be made from Fig. 9. According to the figure, the transversely averaged temperature profile for $\mathrm{Br}=10^{-10}$ is located above the case for $\mathrm{Br}=0$ which indicates a higher temperature for $\mathrm{Br}=10^{-10}$. 


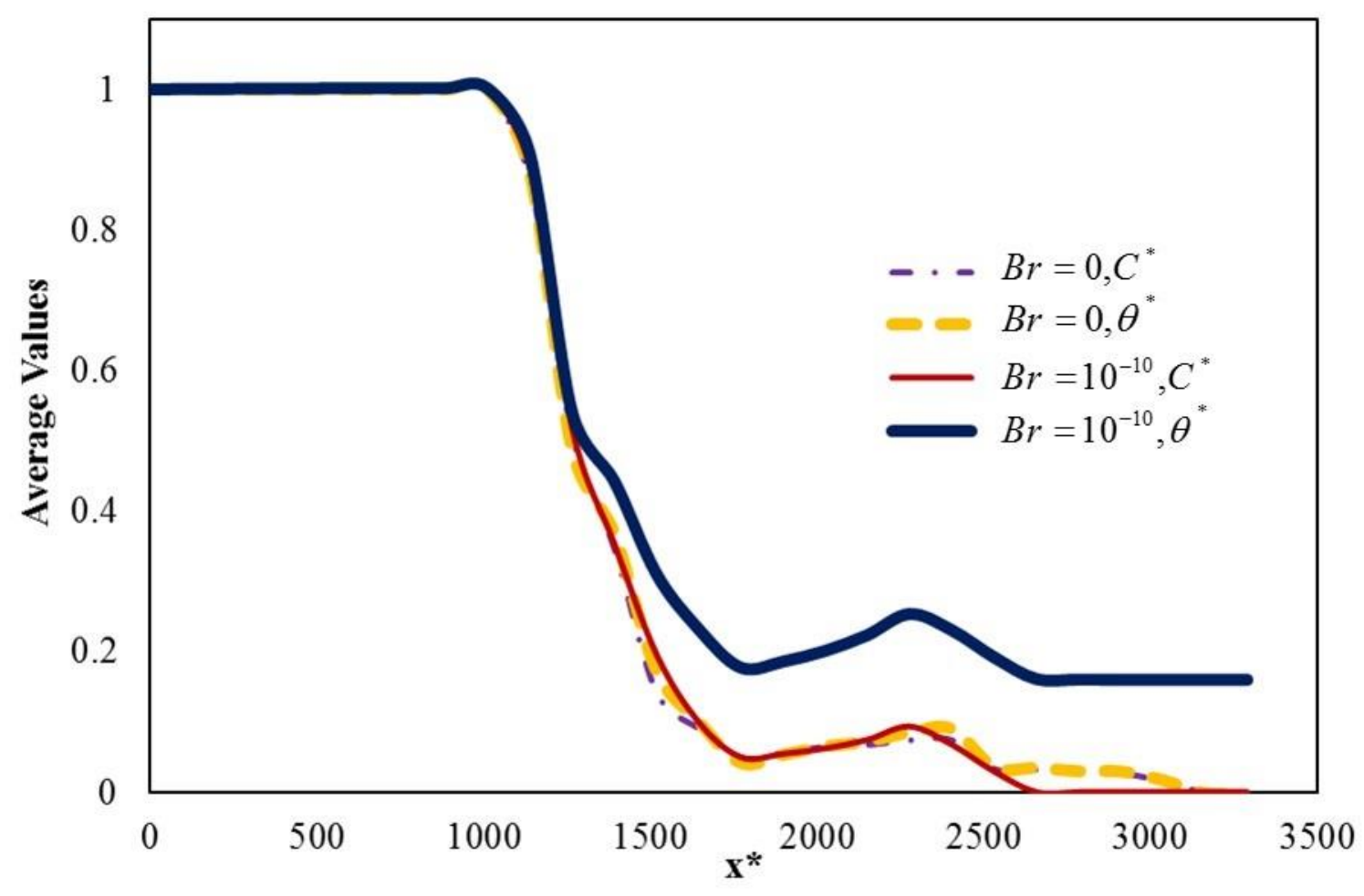

Fig. 13. The effect of viscous dissipation on the transversely averaged concentration and temperature profiles at $t^{*}=640(L e=\lambda=1)$

Figure 14 shows the variation of the transversely averaged concentration and temperature profiles as a function of the thermal lag coefficient $(\lambda)$. The curves apparently have fewer distortions for lower values of thermal lag coefficient i.e. the flow becomes more stable by decreasing this parameter. The transversely averaged temperature profiles show that the increase in fluid temperatures is less for the lower value of $\lambda$. This is due to an increase in heat transfer between the solid and the fluid phases at lower values of the thermal lag coefficient. Additionally, by comparing the diagrams of temperature and concentration, the thermal delay can be observed distinctly. This delay is more obvious at $\lambda=0.5$. The transversely averaged concentration and temperature profiles for different Lewis numbers are shown in Figures 15 and 16, respectively. The curves become smoother by increasing Le and therefore, it can be concluded that the flow becomes more stable at higher values of Lewis number. Additionally, increasing the temperature of fluids resulting from viscous dissipation is less at large Lewis numbers. 


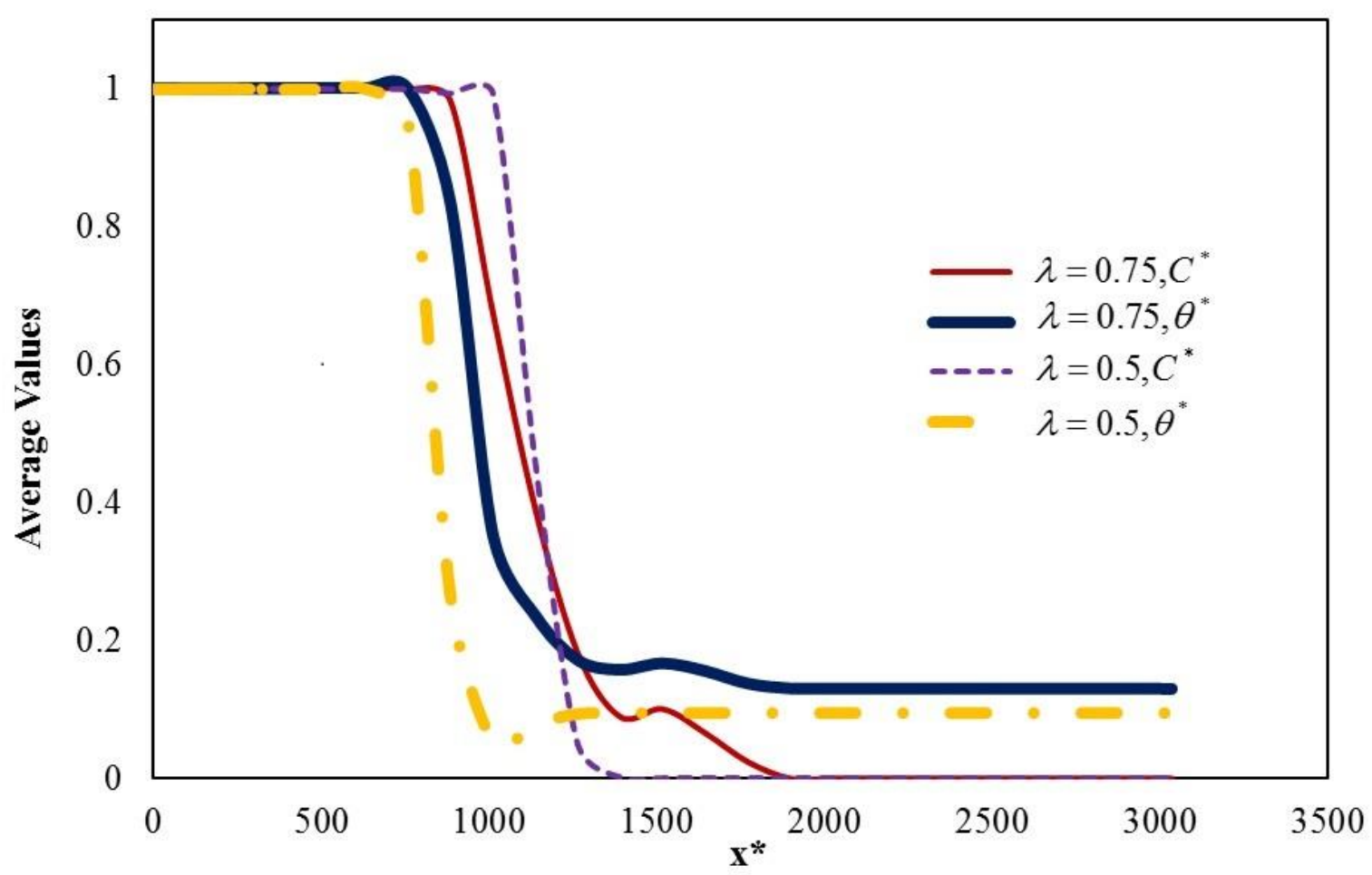

Fig. 14 The effect of thermal lag coefficient on the transversely averaged concentration and temperature profiles at $t^{*}=640\left(\mathrm{Le}=1\right.$ and $\left.B r=10^{-10}\right)$

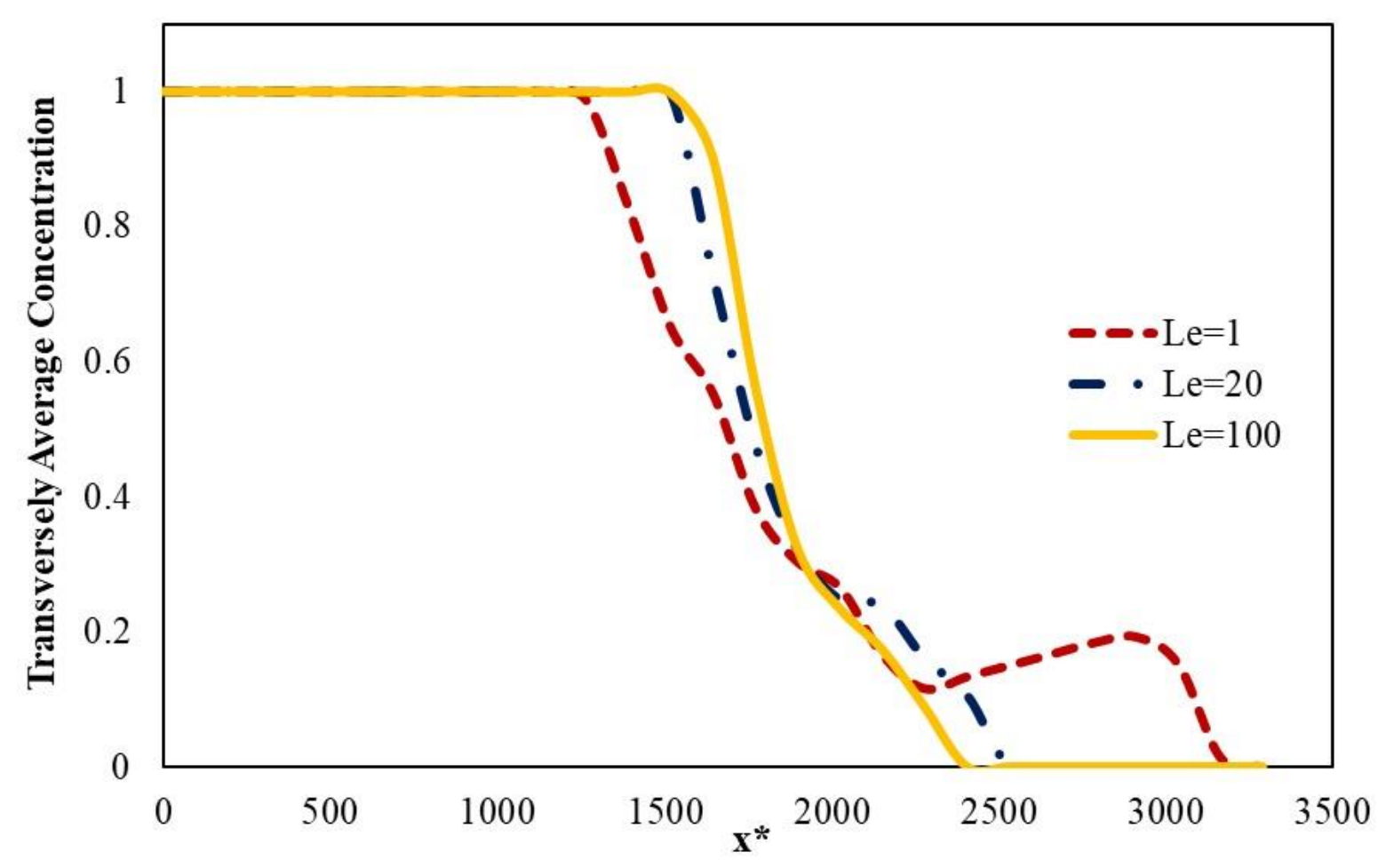

Fig. 15 The effect of Lewis number on the transversely averaged concentration profiles at $t^{*}=640\left(\lambda=0.75\right.$ and $\left.B r=10^{-10}\right)$ 


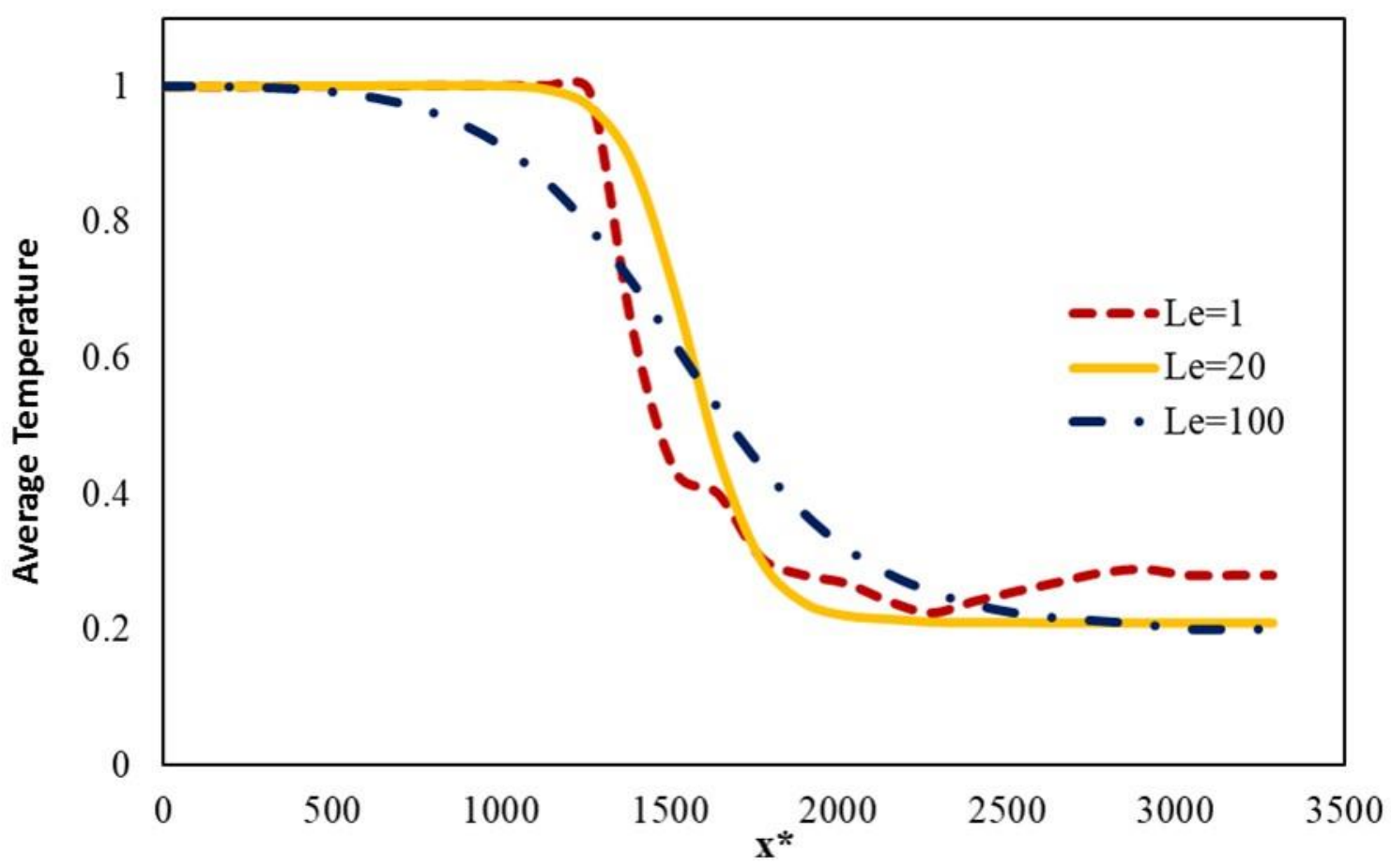

Fig. 16 The effect of Lewis number on the transversely averaged temperature profiles at $t^{*}=640 \quad\left(\lambda=0.75\right.$ and $\left.B r=10^{-10}\right)$

\section{d. Mixing Length}

The degree of penetration of the advancing low viscous fluid through high viscous fluid is called the mixing length. This quantity is used to show the level of progressive movement of fingers through porous media. Indeed, the mixing length shows the length of the longest finger in porous media. In this study, this parameter is measured through the proposed method by Zimmerman and Homsy [27]. In this method, the distance between the specific values of the averaged concentration is considered as the mixing length.

$$
L_{\delta}=x^{*}{ }_{\bar{C}=\delta}-x^{*}{ }_{\bar{C}=1-\delta}
$$

Where, $\delta$ is a small positive number and here it is considered equal to 0.01 .

The variation of mixing length for different values of $B r$ is shown in Figure 17. In the previous sections, it was observed that the viscous dissipation has a stabilizing effect. In other words, the fingers grow at a slower rate for $B r \neq 0$ and penetration through the high viscosity fluid is slower than the case with $B r=0$. Therefore, the mixing length should be lower for $B r \neq 0$. It can be seen that the mixing length decreases by increasing the Brinkman number. The variation of mixing length for different values of $\lambda$ is shown in Fig. 18. A decrease in thermal lag coefficient leads to more stable flow and a reduction in the mixing length. 


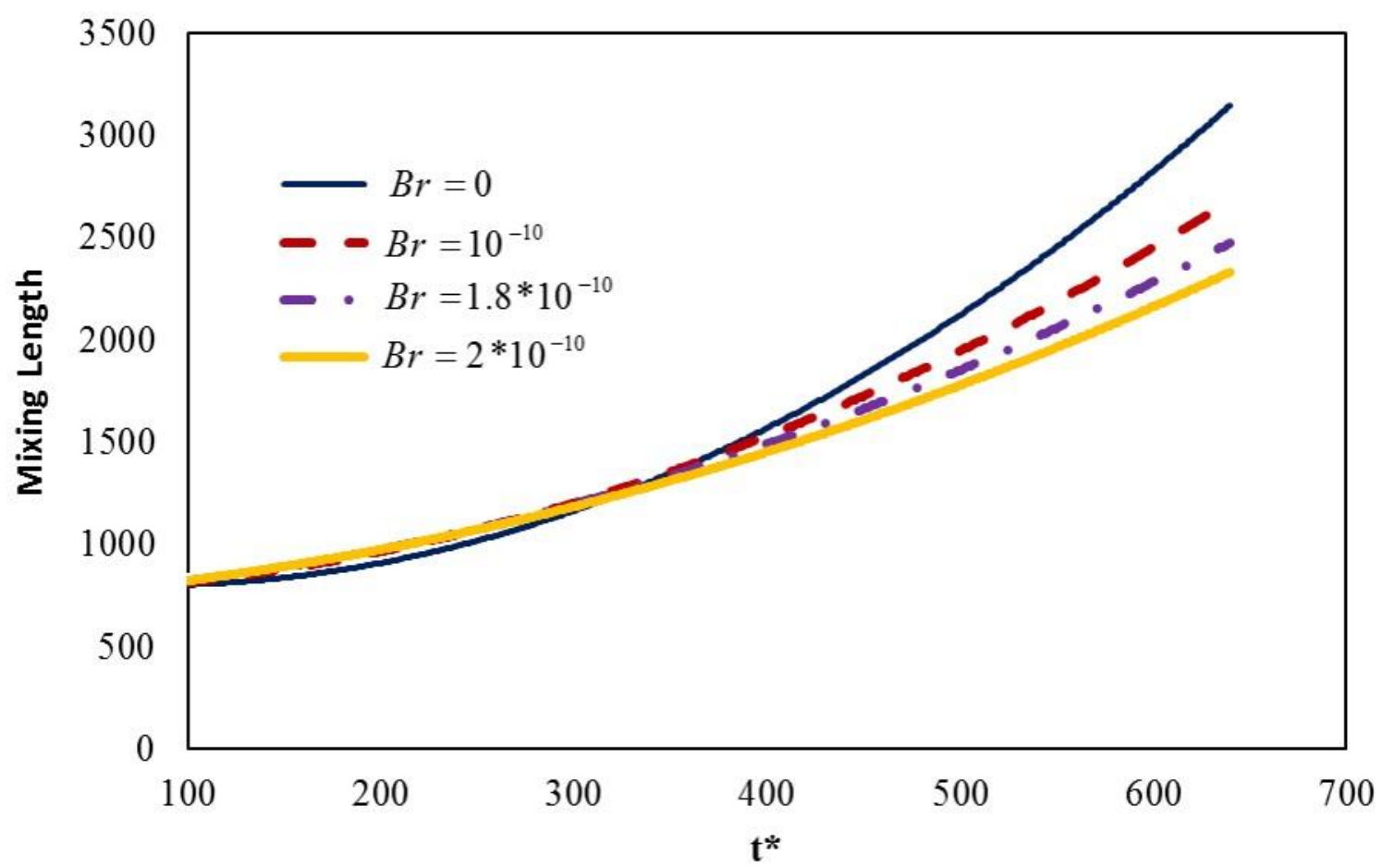

Fig. 17. The effect of Brinkman number on the mixing length $(\lambda=L e=1)$

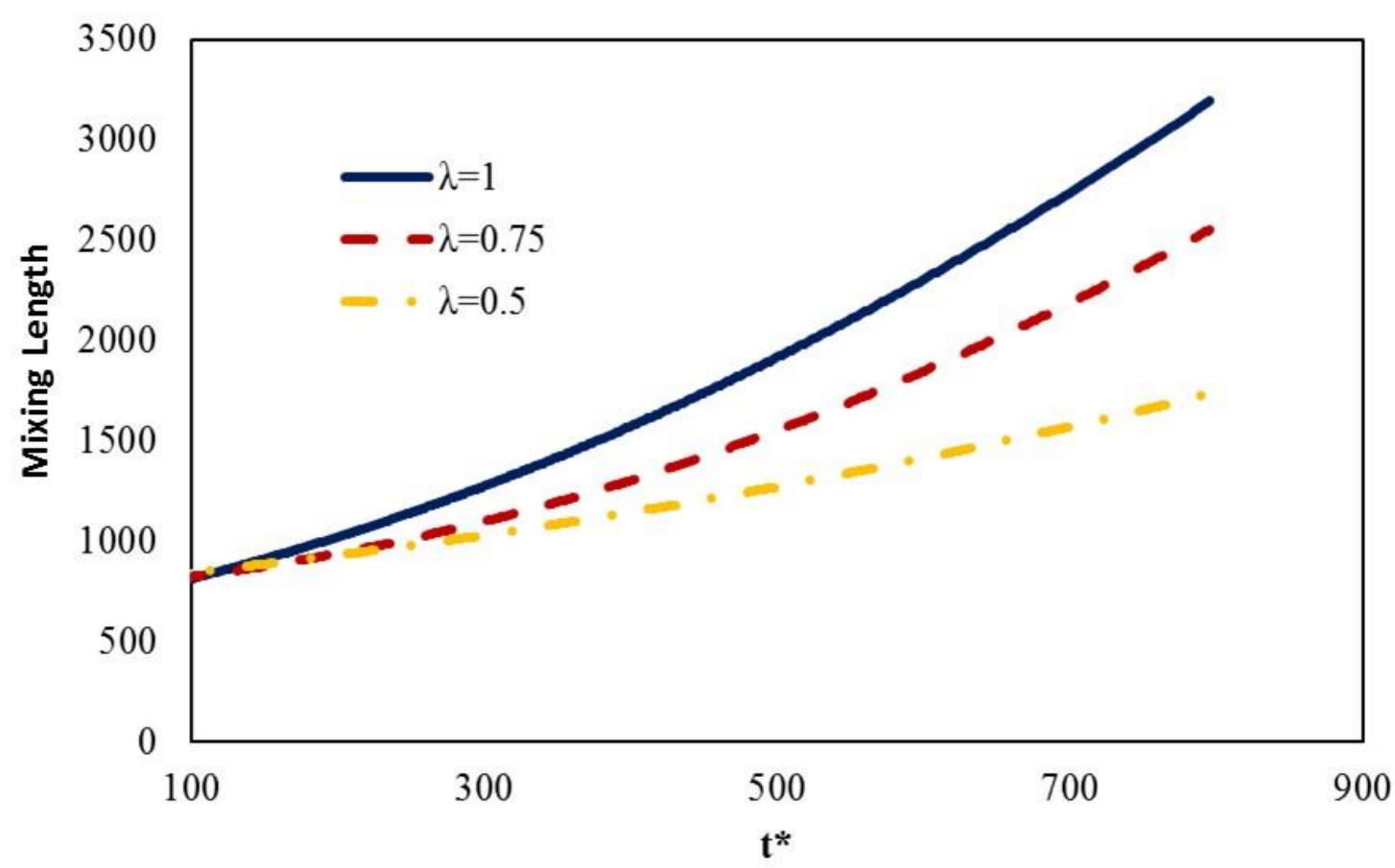

Fig. 18. The effect of thermal lag coefficient on the mixing length $(L e=1)$

Figure 19 shows the distribution of the mixing length versus time for different values of Lewis number. As mentioned before, at lower values of Lewis number, the fingers are generated and 
grow faster. For this reason, the mixing length is also high for small Lewis numbers.

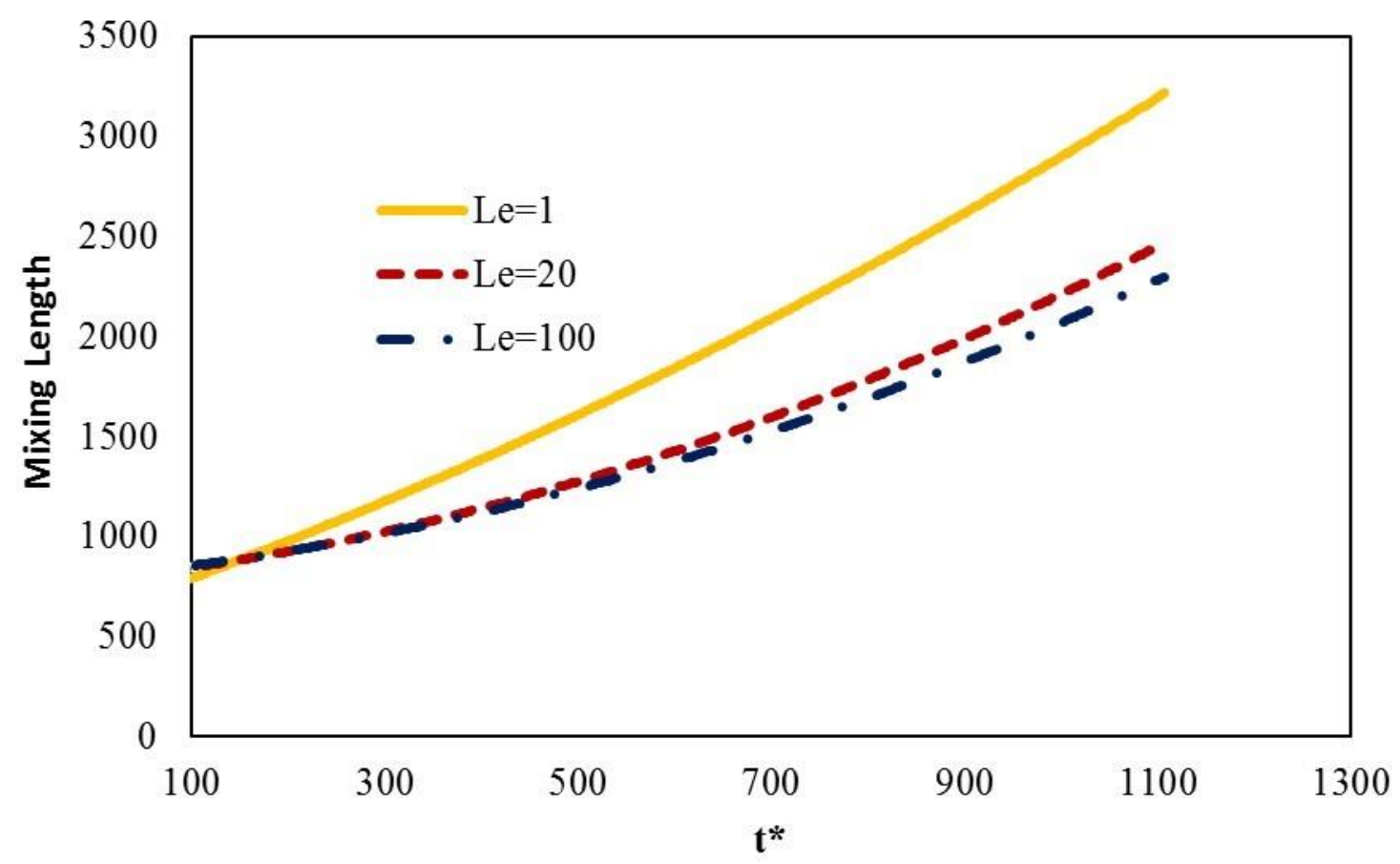

Fig. 19 The effect of Lewis number on the mixing length $(\lambda=0.75)$

\section{e. Sweep efficiency}

The ratio of the volume of displaced fluid to the total volume of available fluid in a porous medium in the displacement process is called the sweep efficiency. There are several alternative definitions for this parameter. In petroleum engineering, sweep efficiency is defined as the ratio of the swept volume of oil by displacing fluid to the total oil in the reservoir and is generally used to quantify the effectiveness of the displacement in enhanced oil recovery process. In this study, this parameter is defined as the ratio of displacing fluid volume to the volume of displaced fluid. The maximum sweep efficiency is obtained when the fingers reach the end of the domain. Following Ghesmat and Azaiez [30], this parameter is defined as:

Sweep efficiency $=$ (the number of grid points where the concentration is equal to or larger than $0.5) /$ (the total number of grid points that are located behind the front defined by $C^{*}=0.5$ )

The variation of sweep efficiency with $\lambda$ for different Brinkman numbers is shown in Figure 20. According to the figure, the sweep efficiency is enhanced by increasing the viscous 
dissipation while increasing the thermal lag coefficient induces the opposite effect. These trends are consistent with the results of temperature and concentration contours in which the level of instability is reduced by increasing $B r$ and decreasing $\lambda$.

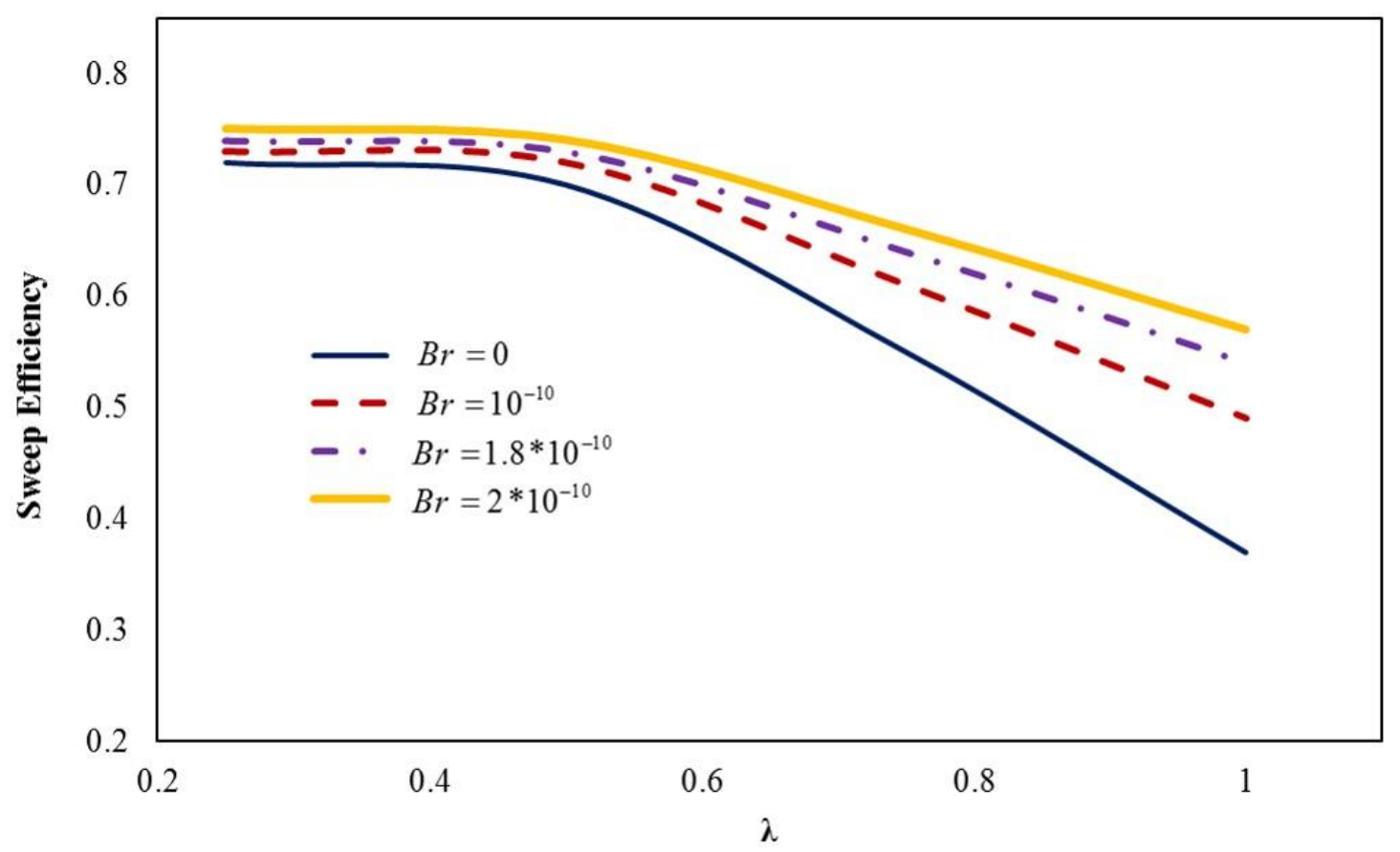

Fig. 20. The effect of Brinkman number on the sweep efficiency ( $L e=1)$

Fig. 21 shows the evolution of the sweep efficiency in terms of thermal lag coefficient for different Lewis numbers. According to the contours of transversely averaged and mixing length, increasing Lewis number and decreasing thermal lag coefficient leads to more stable flow. Therefore, the sweep efficiency increases by increasing Lewis number and decreasing the thermal lag coefficient. 


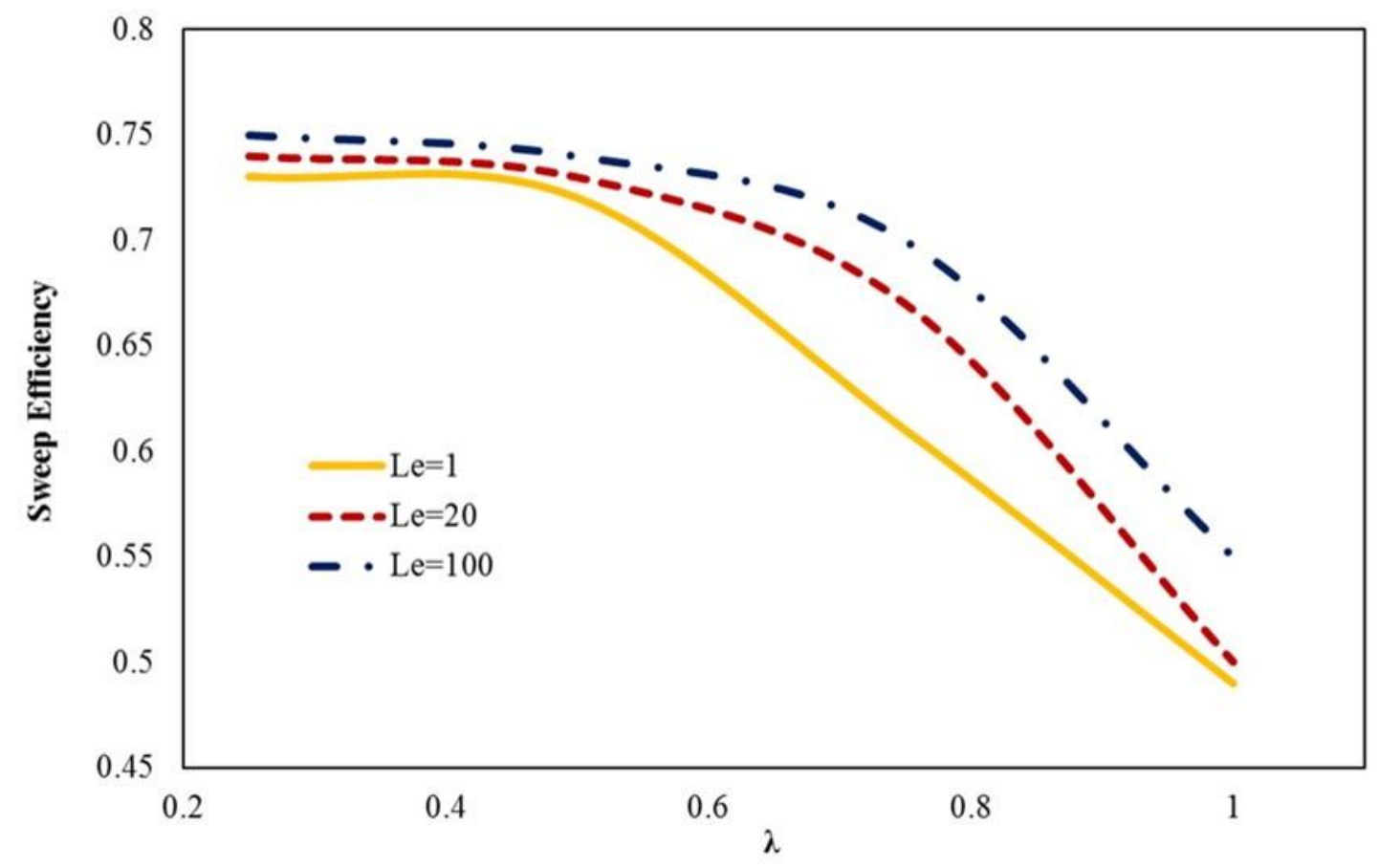

Fig. 21 The effect of Lewis number on the sweep efficiency

\section{f. Breakthrough time}

Sajjadi and Azaiez [20] introduced the breakthrough time as a moment in which the displacing fluid is produced at the production end. In the current study, this parameter represents the time taken for the fingers in the concentration front to reach the end of the domain and it is measured as the time elapsed for the concentration front with $C^{*}=0.01$ to reach the last grid in the longitudinal direction. Figure 22 shows the variation of breakthrough time versus thermal lag coefficient for different Brinkman numbers. According to the figure, viscous dissipation increases the breakthrough time and this increase is larger with greater values of $\lambda$. Since the viscous dissipation increases the temperature and subsequently reduces the viscosity ratio, the flow becomes more stable and therefore, the growth rate of fingers reduces. Additionally, decreasing thermal lag coefficient also leads to an increase in breakthrough time.

The effect of Lewis number on breakthrough time is depicted in Figure 23. As mentioned earlier, the flow becomes more stable by increasing the Lewis number. In light of the interpretation already given, the breakthrough time increases. 


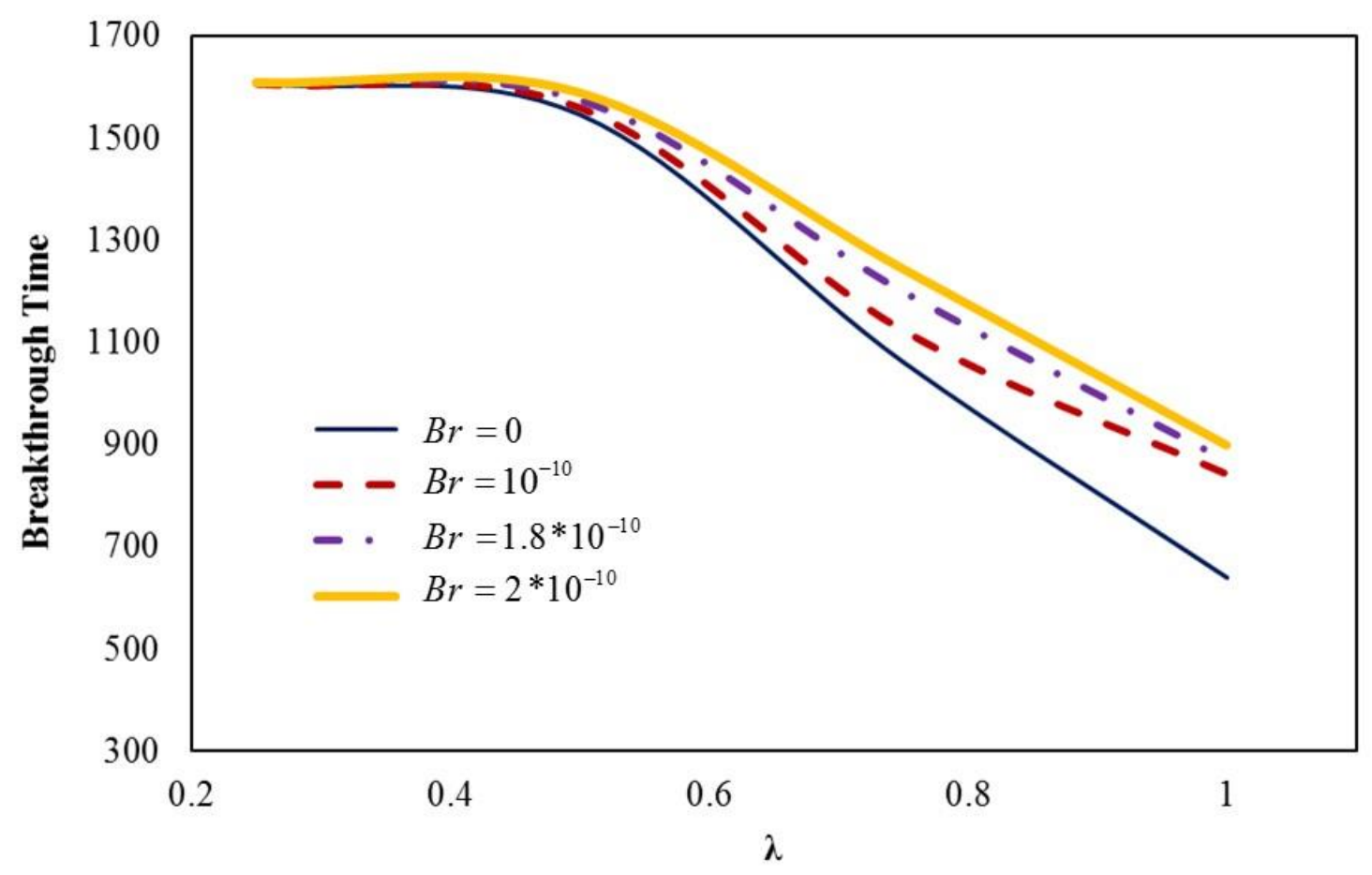

Fig. 22 The effect of Brinkman number on the breakthrough time $(L e=1)$

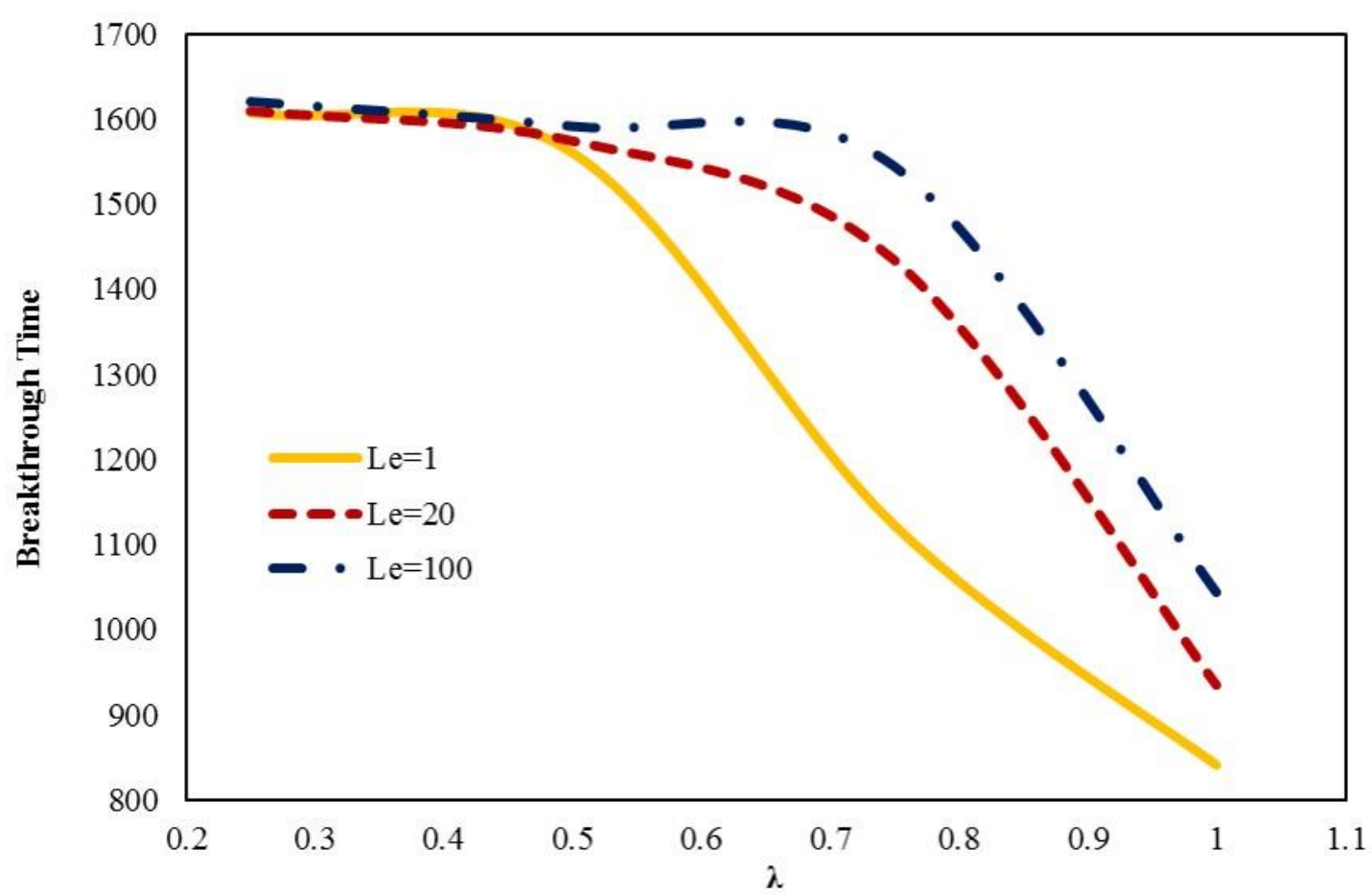

Fig. 23 The effect of the Lewis number on the breakthrough time. 


\section{CONCLUSIONS}

In this study, the thermal viscous fingering instability of miscible displacement through porous media is investigated. Here, it is assumed that a cold high viscosity liquid is displaced by a hot low viscosity one. The non-dimensional conservation equations using a two-phase Darcy formulation are solved with the COMSOL CFD software. The hydrodynamic and thermosolutal characteristics are explored based on the concentration and temperature contours, the averaged concentration and temperature profiles, mixing length and sweep efficiency. The main innovative aspect of the present article is the incorporation of the viscous dissipation effect in thermo-viscous fingering instability simulation. Viscous dissipation acts as a heat source and increases the temperature of both phases. In non-isothermal displacements, due to the viscosity dependence on temperature, viscous dissipation may exert an important role on Saffman-Taylor instability. The results show that the flow is more stable in the presence of viscous dissipation. Additionally, the effect of Lewis number (Le) and thermal lag coefficient $(\lambda)$ are investigated in detail. It is found that increasing Lewis number and decreasing thermal lag coefficient lead to an increase in sweep efficiency and decrease in mixing length. Decreasing $\lambda$ manifests in a boost in the heat transfer between fluids and solid phases and as a result, the velocity of thermal front decreases and it lags behind the concentration front. Thus, the destabilizing effects of the thermal front on the concentration front decreases.

\section{REFERENCES}

[1] D. Bensimon, L. P. Kadanoff, S. Liang, B. I. Shraiman, C. Tang, Viscous flows in two dimensions, Reviews of Modern Physics, Vol. 58, No. 4, pp. 977, 1986.

[2] K. V. McCloud, J. V. Maher, Experimental perturbations to Saffman-Taylor flow, Physics Reports, Vol. 260, No. 3, pp. 139-185, 1995.

[3] S. Hill, Channeling in packed columns, Chemical Engineering Science, Vol. 1, No. 6, pp. 247$253,1952$.

[4] C. Tan, G. Homsy, Stability of miscible displacements in porous media: Rectilinear flow, The Physics of fluids, Vol. 29, No. 11, pp. 3549-3556, 1986.

[5] C. Tan, G. Homsy, Stability of miscible displacements in porous media: Radial source flow, The Physics of fluids, Vol. 30, No. 5, pp. 1239-1245, 1987.

[6] B. K. Singh, J. Azaiez, Numerical simulation of viscous fingering of shear- thinning fluids, The Canadian Journal of Chemical Engineering, Vol. 79, No. 6, pp. 961-967, 2001.

[7] J. Azaiez, B. Singh, Stability of miscible displacements of shear thinning fluids in a Hele-Shaw cell, Physics of Fluids, Vol. 14, No. 5, pp. 1557-1571, 2002.

[8] M. Norouzi, M. Shoghi, A numerical study on miscible viscous fingering instability in anisotropic porous media, Physics of Fluids, Vol. 26, No. 8, pp. 084102, 2014.

[9] M. R. Shoghi, M. Norouzi, Linear stability analysis and nonlinear simulation of non-Newtonian viscous fingering instability in heterogeneous porous media, Rheologica Acta, Vol. 54, No. 1112, pp. 973-991, 2015. 
[10] H. Shokri, M. Kayhani, M. Norouzi, Nonlinear simulation and linear stability analysis of viscous fingering instability of viscoelastic liquids, Physics of Fluids, Vol. 29, No. 3, pp. 033101, 2017.

[11] H. Shokri, M. Kayhani, M. Norouzi, Saffman-Taylor instability of viscoelastic fluids in anisotropic porous media, International Journal of Mechanical Sciences, Vol. 135, pp. 1-13, 2018.

[12] X. Kong, M. Haghighi, Y. Yortsos, Visualization of steam displacement of heavy oils in a HeleShaw cell, Fuel, Vol. 71, No. 12, pp. 1465-1471, 1992.

[13] M. Saghir, O. Chaalal, M. Islam, Numerical and experimental modeling of viscous fingering during liquid-liquid miscible displacement, Journal of Petroleum Science and Engineering, Vol. 26, No. 1-4, pp. 253-262, 2000.

[14] T. Sheorey, K. Muralidhar, P. P. Mukherjee, Numerical experiments in the simulation of enhanced oil recovery from a porous formation, International journal of thermal sciences, Vol. 40, No. 11, pp. 981-997, 2001.

[15] K. E. Holloway, J. R. De Bruyn, Viscous fingering with a single fluid, Canadian journal of physics, Vol. 83, No. 5, pp. 551-564, 2005.

[16] J. Kuang, T. Maxworthy, The effects of thermal diffusion on miscible, viscous displacement in a capillary tube, Physics of Fluids, Vol. 15, No. 5, pp. 1340-1343, 2003.

[17] D. Pritchard, J. A. Pearson, Viscous fingering of a thixotropic fluid in a porous medium or a narrow fracture, Journal of non-newtonian fluid mechanics, Vol. 135, No. 2, pp. 117-127, 2006.

[18] M. Islam, J. Azaiez, Miscible thermo-viscous fingering instability in porous media. Part 1: Linear stability analysis, Transport in porous media, Vol. 84, No. 3, pp. 821-844, 2010.

[19] M. Islam, J. Azaiez, Miscible thermo-viscous fingering instability in porous media. Part 2: Numerical simulations, Transport in porous media, Vol. 84, No. 3, pp. 845-861, 2010.

[20] J. Azaiez, M. Sajjadi, Stability of double-diffusive double-convective miscible displacements in porous media, Physical Review E, Vol. 85, No. 2, pp. 026306, 2012.

[21] M. Sajjadi, J. Azaiez, Dynamics of fluid flow and heat transfer in homogeneous porous media, The Canadian Journal of Chemical Engineering, Vol. 91, No. 4, pp. 687-697, 2013.

[22] S. Jackson, H. Power, D. Giddings, Immiscible thermo-viscous fingering in Hele-Shaw cells, Computers \& Fluids, 2017.

[23] A. Al-Hadhrami, L. Elliott, D. Ingham, A new model for viscous dissipation in porous media across a range of permeability values, Transport in Porous media, Vol. 53, No. 1, pp. 117-122, 2003.

[24] T. S. Lundgren, Slow flow through stationary random beds and suspensions of spheres, Journal of Fluid Mechanics, Vol. 51, No. 2, pp. 273-299, 1972.

[25] J. Rubinstein, Effective equations for flow in random porous media with a large number of scales, Journal of Fluid Mechanics, Vol. 170, pp. 379-383, 1986.

[26] L. Durlofsky, J. Brady, Analysis of the Brinkman equation as a model for flow in porous media, The Physics of fluids, Vol. 30, No. 11, pp. 3329-3341, 1987.

[27] M. Kaviany, Principles of heat transfer in porous media: Springer Science \& Business Media, 2012.

[28] C. Tan, G. Homsy, Simulation of nonlinear viscous fingering in miscible displacement, The Physics of fluids, Vol. 31, No. 6, pp. 1330-1338, 1988.

[29] W. Zimmerman, G. Homsy, Nonlinear viscous fingering in miscible displacement with anisotropic dispersion, Physics of Fluids A: Fluid Dynamics, Vol. 3, No. 8, pp. 1859-1872, 1991.

[30] K. Ghesmat, J. Azaiez, Viscous fingering instability in porous media: effect of anisotropic velocity-dependent dispersion tensor, Transport in Porous Media, Vol. 73, No. 3, pp. 297-318, 2008.

\section{ACKNOWLEDGEMENTS}

The authors are grateful to both reviewers for their insightful comments which have served to significantly improve the present work. 